\title{
The Hamiltonian Structure of General Relativistic Perfect Fluids
}

\author{
David $\mathrm{Bao}^{1 \star}$, Jerrold Marsden ${ }^{2 \star \star}$ and Ronald Walton ${ }^{3}$ \\ 1 School of Mathematics, The Institute for Advanced Study, Princeton, NJ 08540, USA and \\ Department of Mathematics, University of Houston-University Park, Houston, TX 77004, USA \\ 2 Department of Mathematics, University of California, Berkeley, CA 94720, USA \\ 3 Space Systems Division, Lockheed Missiles \& Space Company, Inc., 1111 Lockheed Way, Sunnyvale, \\ CA 94086, USA
}

\begin{abstract}
We show that the evolution equations for a perfect fluid coupled to generai relativity in a general lapse and shift, are Hamiltonian relative to a certain Poisson structure. For the fluid variables, a Lie-Poisson structure associated to the dual of a semi-direct product Lie algebra is used, while the bracket for the gravitational variables has the usual canonical symplectic structure. The evolution is governed by a Hamiltonian which is equivalent to that obtained from a canonical analysis. The relationship of our Hamiltonian structure with other approaches in the literature, such as Clebsch potentials, Lagrangian to Eulerian transformations, and its use in clarifying linearization stability, are discussed.
\end{abstract}

\section{Introduction}

The purpose of this paper is to study the Hamiltonian nature of the Einstein-Euler field equations, which govern the "Eulerian" description of general relativistic perfect fluids. We show that the corresponding evolution equations, when written in a general lapse and shift, are Hamiltonian with respect to a Poisson bracket given by the Dirac-ADM canonical Poisson bracket for the gravitational variables (Dirac, 1959, Arnowitt et al., 1962) and by a "Lie-Poisson" bracket for the fluid variables.

A Lie-Poisson bracket is a noncanonical Poisson bracket defined by a Lie algebraic structure associated with the symmetries of a Hamiltonian system. For non-relativistic fluids, these brackets are due to Morrison and Greene (1980) and Dzyaloshinski and Volovik (1980). The group theoretic origin of Lie-Poisson brackets for Hamiltonian systems and their derivation from canonical brackets in

* Research supported in part by NSF grant MCS 81-08814(A02)

$\star \star$ Research supported in part by NSF grant MCS 81-07086 
Lagrangian coordinates by reduction is due to Arnold (1966), Marsden and Weinstein (1982, 1983), Marsden et al. (1983) and Marsden et al. (1984a). The fact that the brackets for nonrelativistic compressible fluids are Lie-Poisson brackets for a semidirect product Lie algebra was well-known by 1980 and is an instance of the general results of Ratiu (1980) and Guillemin and Sternberg (1980). This fact was shown to also hold in magnetohydrodynamics and other systems by Holm and Kupershmidt (1983). In these papers, and in references cited therein, the equations for the Eulerian descriptions of plasma physics, magnetohydrodynamics, elasticity theory, and other theories from classical continuum mechanics are also shown to be Hamiltonian with respect to brackets closely associated to Lie-Poisson brackets. It is therefore not surprising that the Poisson bracket for the Hamiltonian structure of general relativistic perfect fluids involves a Lie-Poisson bracket for the fluid variables.

The Lie-Poisson formalism automatically builds in the "Lin constraints," conditions needed for a variational principle in the Eulerian picture; see Seliger and Witham (1968) and Bretherton (1970). The Lin constraints are exactly the coadjoint orbits - constraint manifolds naturally associated with the Lie-Poisson structure (see, for instance, Marsden et al. 1983).

Other Hamiltonian structures have been proposed for the Einstein-Euler field equations which differ from the one presented in this paper. The original variational principles for relativistic fluids, based on varying the particles' world lines, are due to Taub (1948). (See Misner et al. 1973 for further references). Schutz (1970, 1971) has proposed a canonical Hamiltonian structure based on the use of nonphysical Clebsch potentials. (The relationship between the Lie-Poisson approach and Clebsch potentials is described in Holm and Kupershmidt (1983) and in Marsden and Weinstein (1983); see Sect. 5 below.) Moncrief $(1974,1977)$ has proposed a canonical Hamiltonian structure in which the spacetime coordinates are tied to the fluid by a set of coordinate conditions introduced by Taub (1969), thereby allowing Moncrief to formally eliminate the explicit appearance of any independent fluid variables. Walton $(1979,1980)$ proposed a noncanonical Hamiltonian structure which also has the unique feature that it uses a parametrization of the general relativistic gauge algebra relating the spacetime coordinates to the streamlines of the fluid rather than to the normal congruence of the spacelike slicing of spacetime. The relationship between these "other" Hamiltonian structures for general relativistic perfect fluids and the one presented in this paper will be discussed in the final section of this paper.

One of the features of this paper is to establish a Hamiltonian formalism which allows an arbitrary gauge or coordinate condition. Indeed, in some numerical work it is not always desirable to use comoving coordinates; see Smarr et al. (1980).

Moncrief (1974) has used his Hamiltonian structure for general relativistic perfect fluids to investigate the stability of spherically symmetric neutron star cores. Walton (1979) used his Hamiltonian structure to investigate adiabatic linear perturbations of spatially-flat, Robertson-Walker universes, Lund (1973) used Schutz's Hamiltonian structure to quantize the dynamics of a spherically symmetric, gravitationally collapsing star. The Hamiltonian structure for the Einstein-Euler field equations presented in this paper should also find applications in astrophysics 
and cosmology as well as in other more theoretical investigations of the EinsteinEuler system. Among the latter applications we include the linearization stability problem which is posed by the question: when is a solution of the linearized Einstein-Euler field equations tangent to a curve of exact solutions?

We believe that if the perturbations are confined to Lin constraint surfaces (the coadjoint orbits) then the basic results connecting symmetry with linearization instabilities and singularities in the space of solutions hold (Fischer et al. 1980; Arms et al., 1981, 1982 and references therein). However, if perturbations off the coadjoint orbits are allowed, this link is destroyed (D'Eath, 1974 and Arms, 1979*).

A second application is to the study of dynamic stability of equilibrium solutions. Since the Poisson bracket structure is the same as in the nonrelativistic case, the methods used there should carry over (see, for example, Arnold, 1966b; Holm et al. 1983, 1984; Abarbanel et al., 1984).

Finally, we mention that the generalization of our work to the case of general relativistic plasmas, MHD and elasticity now seems straightforward, given the nonrelativistic literature available (cf. Bialynicki-Birula et al. 1982; Carter, 1973; Spencer, 1982; Tam, 1966; Tam and O'Hanlon, 1969; Taub, 1970; Holm and Kupershmidt, 1984).

This paper is organized into five sections. In Sect. 1 we review preliminaries including some facts about equations of state for perfect fluids, the kinematics of spacelike slicings of spacetimes containing perfect fluids, and some results about Lie-Poisson brackets which are essential to this paper. Section 2 reviews the Einstein-Euler equations and also gives a $(3+1)$-decomposition of these equations using the usual lapse function and shift vector field parametrization of the general relativistic gauge algebra. In Sect. 3 we derive the main result of this paper, a Hamiltonian structure for the $(3+1)$-decomposed Einstein-Euler equations of Sect. 2 in which the Hamiltonian function is parametrized by the lapse function and the shift vector field in the usual Dirac (1964) form. Then, in Sect. 4, we introduce another Hamiltonian function for the Einstein-Euler equations which is parametrized by Walton's (1980) generalized lapse function and shift vector field relating the spacetime coordinates to the streamlines of the fluid rather than to the normal congruence of the spacelike slicing of spacetime. Finally, in Sect. 5, we discuss the relationship between the Hamiltonian structure for general relativistic perfect fluids presented in this paper and those of Schutz, Moncrief, and Walton. We also elaborate on the Lagrangian to Eulerian transformations (including the possibility of using fluid variables analogous to van Dantzig's (1939) thermasy function - a variable "conjugate" to the entropy) based on Marsden et al. (1984b).

\section{Preliminaries}

A. The Equation of State and some Thermodynamic Parameters. The equation of state of a fluid is usually given by the energy (per unit rest space 3-volume)

$$
e=e(n, s)
$$

as a function of the baryon number (per unit rest space 3-volume) $n$ and the entropy (per baryon, in a rest frame) $s$, or given by the isotropic pressure (in a 
rest frame)

$$
p=p(h, s)
$$

as a function of $s$ and the enthalpy (per baryon, in a rest frame) $h$. The quantities $e, p, n, h$ are related by

$$
n h=e+p \text {. }
$$

In Misner et al. (1973), p. 564, one learns that $e+p$ (equivalently $n h$ ) can be given the interpretation of a relativistic inertial mass (per unit rest space 3-volume). We will, on two occasions, need the physically reasonable assumption that

$$
n h \neq 0 \text {. }
$$

We shall assume that energy is conserved in the fluid's rest frame. This assumption is formalized as the first law of thermodynamics, which takes on the following equivalent forms

$$
\begin{aligned}
& d e=h d n+n T d s \\
& d p=n d h-n T d s
\end{aligned}
$$

where

$$
T=n^{-1} \partial_{s} e=-n^{-1} \partial_{s} p
$$

is the temperature (in the rest frame), and $\partial_{s}$ denotes partial differentiation.

From the above we see that

$$
h=\partial_{n} e, \quad p=n \partial_{n} e-e,
$$

and

$$
n=\partial_{h} p, \quad e=h \partial_{h} p-p .
$$

Thus $e$ and $p$ are related by Legendre transformations, while $n$ and $h$ are thermodynamically conjugate variables. This demonstrates the equivalence between the equations of state (1A.1) and (1A.2). We shall use (1A.2) as our equation of state.

The quantity $v$ defined by

$$
v^{-2}=h n^{-1} \partial_{h} n
$$

is the speed of sound in the fluid, as measured in the rest frame (see Taub, 1948; Lichnerowicz, 1967). We will on two later occasions need the causality assumption

$$
v \leqq 1
$$

where 1 is the speed of light in vacuum, given in geometrical units.

$B$. Slicings, the 4-Vector $J$, the Lapse and Shift. Our spacetime $X$ is a 4-dimensional Lorentzian manifold with signature $(-+++)$. We assume that $X$ is topologically $\Sigma \times \mathbb{R}$, where $\Sigma$ is a compact, oriented, boundaryless 3-dimensional manifold. The compact-boundaryless assumption is made to facilitate integration by parts; it may be replaced by asymptotic conditions on functions at spatial infinity. 
A slicing is a curve of embeddings $i_{t}: \Sigma \rightarrow X$. The tangent to this curve is the evolution vector field $\partial_{t}$. The slicing, viewed as $i: \Sigma \times \mathbb{R} \rightarrow X$, provides a local coordinate system $\left\{x^{\mu}\right\}$ on $X$ (whenever $i$ is a diffeomorphism onto its image). A local coordinate basis is provided by $\left\{\partial_{t}, i_{t} \partial_{i}\right\}$, where $\left\{\partial_{i}\right\}$ constitutes a local coordinate basis on $\Sigma$.

Our index conventions are as follows: $\mu, v, \ldots$ (lower case Greek) are spacetime coordinate indices, they run from 0 to 3 and are raised and lowered with the 4-metric ${ }^{(4)} g=g_{\mu v} d x^{\mu} \otimes d x^{v} ; i, j, \ldots$ (lower case Latin) are coordinate indices on the hypersurface $\Sigma$, they run from 1 to 3 and are raised and lowered with the induced time-dependent 3-metric $g=i_{t}^{*(4)} g=g_{i j} d x^{i} \otimes d x^{j}$. Vector indices are up, co-vector indices are down; repeated indices are automatically summed. The superscript \# on a tensor means that the later's indices are all raised; likewise, the superscript $b$ on a tensor means that its indices are all lowered. Covariant differentiation on spacetime will be denoted by ${ }^{(4)} \nabla$ or ";", and that on $\Sigma$ will be denoted by $\nabla$ or "". Our definition of the 4-curvature is

$$
R_{\beta \mu \nu}^{\alpha}=\partial_{\mu}\left\{\left\{_{\beta \nu}^{\alpha}\right\}-\partial_{v}\left\{\begin{array}{c}
\alpha \\
\beta \mu
\end{array}\right\}+\left\{\begin{array}{c}
\alpha \\
\xi \mu
\end{array}\right\}\left\{\begin{array}{c}
\xi \\
\beta \nu
\end{array}\right\}-\left\{\begin{array}{c}
\alpha \\
\xi \nu
\end{array}\right\}\left\{\begin{array}{c}
\xi \\
\beta \mu
\end{array}\right\},\right.
$$

where \{\} are the Christoffel symbols of the torson-free connection ${ }^{(4)} \nabla$. The Ricci, scalar, and Einstein curvature tensors are, respectively,

$$
\operatorname{Ric}_{\mu \nu}=R_{\mu \xi \nu}^{\xi}\left(=R_{\mu}{ }_{\nu \xi}^{\xi}\right), \quad{ }^{(4)} R=\operatorname{Ric}_{\xi}^{\xi}, \quad \operatorname{Ein}_{\mu \nu}=\operatorname{Ric}_{\mu \nu}-\frac{1}{2} g_{\mu \nu}{ }^{(4)} R .
$$

The definition of 3-curvature tensors are analogous.

Our basic fields are as follows. The gravitational field is described by the 4-metric ${ }^{(4)} g$. Let $u$ denote the unit 4-velocity of the fluid's flow lines; $u$ is future-pointing, timelike and satisfies

$$
u^{v} u_{v}=-1
$$

The fluid's motion is determined by the vector field

$$
J=u h^{-1},
$$

together with the entropy (per baryon in rest space) $s$. In fact, if $J$ were known, then using (1B.1), so is $h$ (and hence $u$ ); using $h$ and $s$, one can then determine all other thermodynamic quantities from the equation of state (1A.2).

We recall from Lichnerowicz (1967) that the stream lines of an isentropic perfect fluid are geodesics of the conformal spacetime metric

$$
{ }^{(4)} \tilde{g}={ }^{(4)} g h^{2} \text {, }
$$

so that

$$
\mathscr{L}_{J}\left(h^{2} J^{b}\right)=0,
$$

where $\mathscr{L}_{J}$ denotes the Lie differentiation along $J$.

Let $n$ denote the unit normal vector field on the embedded hypersurface $i_{t} \Sigma$. (There is no occasion in this paper where a notational confusion with the baryon number arises.) For a vector field $A$ on spacetime, we use the notation

$$
A_{\perp}=A^{v} n_{v},
$$


and

$$
A^{\perp}=-A_{\perp}=-A^{v} n_{v} .
$$

Using the decomposition

$$
J=J^{\perp} n+i_{t^{\star}}\left(J_{\|}^{k} \partial_{k}\right)
$$

and (1B.1, 1B.2), we get

$$
-h^{-2}=-\left(J^{\perp}\right)^{2}+\left(J_{\|}\right)^{2},
$$

with the abbreviation

$$
\left(J_{\|}\right)^{2}=J_{\|}^{i} J_{\|}^{j} g_{i j} .
$$

As a result, we have $h=h\left(J^{\perp}, J_{\|}, g\right)$. Here, $J^{\perp}$ and $J_{\|}$are, respectively, a timedependent scalar field and a time-dependent vector field on $\Sigma$. Two trivial but useful consequences of (1B.8) are

$$
J^{\perp} \neq 0
$$

and

$$
\left(J_{\|}\right)^{2}\left(J^{\perp}\right)^{-2}<1 .
$$

While we do not dispute the physical significance of $\left(J^{\perp}, J_{\|}\right)$, we shall soon see that the structure of the evolution equations of the Einstein-Euler system becomes more transparent by the use of a different set of physically meaningful fluid variables.

The usual lapse function $N$ and shift vector field $M$ on $\Sigma$ are defined by

$$
N n+i_{t^{\star}} M=\partial_{t} \text {. }
$$

It will also be useful to define the time-dependent scalar field $\bar{N}$ and the time-dependent vector field $\bar{M}$ on $\Sigma$ by the decomposition

$$
\bar{N} J-i_{t^{\star}} \bar{M}=\partial_{t} \text {. }
$$

One can verify that

$$
\bar{N}=N\left(J^{\perp}\right)^{-1}, \quad N=\bar{N} J^{\perp}
$$

and

$$
\bar{M}=-M+N J_{\|}\left(J^{\perp}\right)^{-1}, \quad M=-\bar{M}+\bar{N} J_{\|} .
$$

If one interprets the slicing as reference frames used by a collection of observers, then $\bar{N}$ fixes the clock rates of our observers relative to proper time along $J$ (measured with respect to the conformal spacetime metric (1B.3)), and $\bar{M}$ represents the velocity of the fluid relative to our observers. This interpretation of $\bar{M}$ is one justification for the use of the minus sign in (1B.13). (The variables $J$ and the fluid based lapse and shift $\bar{N}$ and $\bar{M}$ were used in Walton, 1980).

C. Lie-Poisson Structures. The material presented in this section is a condensed and specialized version of that in Marsden et al. (1984a).

Let us first describe the semi-direct product group $S={ }^{\left({ }^{3}\right)} \mathscr{D} \propto\left(\mathscr{F} \times \mathscr{F}^{*}\right)$. $\operatorname{Let}^{\left({ }^{(3)} \mathscr{D}\right.}$ be the diffeomorphism group on $\Sigma, \mathscr{F}$ the space of functions on $\Sigma$, and $\mathscr{F} *$ the 
space of scalar densities on $\Sigma\left(\xi \in \mathscr{F}^{*}\right.$ has the form $\xi=f \sqrt{g}$, where $f$ is a function on $\Sigma$ and $\left.\sqrt{g}=\left(\operatorname{det} g_{i j}\right)^{1 / 2}\right)$. We let ${ }^{(3)} \mathscr{D}$ act by the push-forward action $\rho$ on both $\mathscr{F}$ and $\mathscr{F}^{*}$, namely

$$
\rho(\sigma) \cdot f=f \circ \sigma^{-1},
$$

and

$$
\rho(\sigma) \cdot(f \sqrt{g})=\left(f \circ \sigma^{-1}\right)(\operatorname{det} D \sigma)^{-1} \sqrt{g},
$$

where $D \sigma$ is the Frechet derivative of the diffeomorphism $\sigma: \Sigma \rightarrow \Sigma$. Group multiplication on $S$ is given by

$$
\left(\sigma_{1}, f_{1}, \xi_{1}\right)\left(\sigma_{2}, f_{2}, \xi_{2}\right)=\left(\sigma_{1} \sigma_{2}, f_{1}+\rho\left(\sigma_{1}\right) \cdot f_{2}, \xi_{1}+\rho\left(\sigma_{1}\right) \cdot \xi_{2}\right)
$$

The Lie algebra of $S$ is $\jmath=\mathscr{X} \propto\left(\mathscr{F} \times \mathscr{F}^{*}\right)$, where $\mathscr{X}$ is the space of vector fields on $\Sigma$; i.e. $\mathscr{X}$ is the Lie algebra of ${ }^{(3)} \mathscr{D}$ with the left Lie bracket $[X, Y]:=[X, Y]_{L}=$ $\mathscr{L}_{Y} X$ (which is minus the standard Lie bracket of vector fields). The action $\rho^{\prime}$ of $\mathscr{X}$ on $\mathscr{F}$ and $\mathscr{F}^{*}$ is the linearization of $\rho$ and is given by

$$
\rho^{\prime}(X) \cdot f=-\mathscr{L}_{X} f
$$

(if $\phi_{t}$ is the flow of $X$, then $\rho^{\prime}(X) \cdot f=d /\left.d t\right|_{t=0} \rho\left(\phi_{t}\right) \cdot f=d /\left.d t\right|_{t=0} f \circ \phi_{-t}=$ $\left.-(d f)(X)=-\mathscr{L}_{X} f\right)$; similarly

$$
\rho^{\prime}(X) \cdot(f \sqrt{g})=-\mathscr{L}_{X}(f \sqrt{g})
$$

The Lie bracket on $s$ is

$$
\begin{aligned}
{\left[\left(X_{1}, f_{1}, \xi_{1}\right),\left(X_{2}, f_{2}, \xi_{2}\right)\right] } & =\left(\left[X_{1}, X_{2}\right], \rho^{\prime}\left(X_{1}\right) \cdot f_{2}\right. \\
& \left.-\rho^{\prime}\left(X_{2}\right) \cdot f_{1}, \rho^{\prime}\left(X_{1}\right) \cdot \xi_{2}-\rho^{\prime}\left(X_{2}\right) \cdot \xi_{1}\right) .
\end{aligned}
$$

We will show in Sects. 3 and 4 that an appropriate choice of phase space for a perfect fluid is $\jmath^{*}=\mathscr{X}^{*} \times \mathscr{F}^{*} \times \mathscr{F}$. We hasten to point out that while this phase space is the dual of the Lie algebra of the group ${ }^{(3)} \mathscr{D} \propto\left(\mathscr{F} \times \mathscr{F}^{*}\right)$, the group (3) $\mathscr{D} \propto \mathscr{F}$ whose Lie algebra is parametrized by the lapse function and shift vector field, plays an important role in terms of gauge symmetries.

The elements of $s^{*}$ will be denoted by $(\mu, \eta, s)$ where $\mu$ is a one-form density (the "inertial" 3-momentum one-form density), $\eta$ is a scalar density (the baryon number scalar density), and $s$ is a scalar (the entropy scalar). A concrete discussion of the physical meaning of $\mu$ and $\eta$ will be given in Sect. 3. Both $\mu$ and $\eta$ are products of a tensor with the 3 -volume $\sqrt{g}$, and the natural pairing between $s *$ and $s$ is

$$
\langle(\mu, \eta, s),(X, f, \xi)\rangle=\int_{\Sigma}\left(\mu_{i} X^{i}+\eta f+s \xi\right) d^{3} x .
$$

We now digress a bit. Any Lie group $G$ acts on its Lie algebra $g$ by the adjoint action $X \mapsto \operatorname{Ad}_{g}(X)$, and acts on $\mathrm{g}^{*}$ by the coadjoint action $\mu \mapsto \operatorname{Ad}_{g}^{*-1}(\mu)$. The orbit through $\mu \in \mathfrak{g}^{*}$ is called a coadjoint orbit, and is denoted $\mathcal{O}$. The easily verified statement $d /\left.d t\right|_{t=0}\left\langle\operatorname{Ad}_{\exp (-t X)}^{*}(\mu), Y\right\rangle=\left\langle-(\operatorname{ad} X)^{*} \mu, Y\right\rangle$ (where ad, the linearization of $\mathrm{Ad}$, is the bracketing action of $\mathfrak{g}$ on $\mathfrak{g}$, i.e. $(\operatorname{ad} X) Y=[X, Y])$ shows that the infinitesimal generator of the action of $G$ on $\mathfrak{g}^{*}$ is the vector field $X_{\mathrm{g}^{*}}$, where $X_{\mathrm{g}^{*}}(\mu)=-(\operatorname{ad} X)^{*} \mu \in T_{\mu} \mathfrak{g}^{*} \approx \mathrm{g}^{*}$. By its definition, $X_{\mathrm{g}^{*}}$ is a vector 
field on $\mathfrak{g}^{*}$, which is tangent to each coadjoint orbit. Furthermore, it is clear that the tangent space $T_{\mu} \mathcal{O}$ is spanned by elements of the form $X_{\mathrm{g}} *(\mu)$, where $X \in \mathfrak{g}$.

For the semi-direct product we are considering, the infinitesimal generator (of the coadjoint action of $S$ on $s^{*}$ ) is

$$
(X, f, \xi)_{,} *(\mu, \eta, s)=-[\operatorname{ad}(X, f, \xi)]^{*}(\mu, \eta, s),
$$

where

$$
[\operatorname{ad}(X, f, \xi)]^{*}(\mu, \eta, s)=\left([\operatorname{ad} X]^{*} \mu-\rho_{f}^{*} \eta-\rho_{\xi}^{*} s, \rho^{\prime}(X)^{*} \eta, \rho^{\prime}(X)^{*} s\right) .
$$

Here $\rho_{f}^{\prime}(Y)=\rho^{\prime}(Y) \cdot f, \quad \rho_{\xi}^{\prime}(Y)=\rho^{\prime}(Y) \cdot \xi \quad$ and $\quad \rho_{f}^{\prime *}, \quad \rho_{\xi}^{\prime *}$ are their adjoints. Integration by parts shows that

$$
[\operatorname{ad}(X, f, \xi)]^{*}(\mu, \eta, s)=\left(\mathscr{L}_{X} \mu+\eta d f-\xi d s, \mathscr{L}_{X} \eta, \mathscr{L}_{X} s\right) .
$$

We remind the reader that if $T$ is a tensor, Lie differentiation of the tensor density $T \sqrt{g}$ is defined as

$$
\mathscr{L}_{X}(T \sqrt{g})=\left(\mathscr{L}_{X} T\right) \sqrt{g}+T X_{\mid k}^{k} \sqrt{g},
$$

where $X^{k}{ }_{\mid k}=X^{k}{ }_{, k}+\left\{{ }_{j k}{ }_{j k}\right\} X^{j}$ is the covariant divergence of $X$ on $\Sigma$. From (1C.8) and (1C.10), we get

$$
(X, f, \xi)_{s^{*}}(\mu, \eta, s)=\left(-\mathscr{L}_{X} \mu-\eta d f+\xi d s,-\mathscr{L}_{X} \eta,-\mathscr{L}_{X} s\right) .
$$

For functions $F, H: \diamond^{*} \rightarrow \mathbb{R}$, we can define two $( \pm)$ Lie-Poisson brackets

$$
\begin{aligned}
\{F, H\}_{ \pm}(\mu, \eta, s)= & \pm\left\langle\mu,\left[\frac{\delta F}{\delta \mu}, \frac{\delta H}{\delta \mu}\right]\right\rangle \\
& \pm\left\langle\eta, \rho^{\prime}\left(\frac{\delta F}{\delta \mu}\right) \cdot \frac{\delta H}{\delta \eta}-\rho^{\prime}\left(\frac{\delta H}{\delta \mu}\right) \cdot \frac{\delta F}{\delta \eta}\right\rangle \\
& \pm\left\langle s, \rho^{\prime}\left(\frac{\delta F}{\delta \mu}\right) \cdot \frac{\delta H}{\delta s}-\rho^{\prime}\left(\frac{\delta H}{\delta \mu}\right) \cdot \frac{\delta H}{\delta s}\right\rangle .
\end{aligned}
$$

The functional derivatives of, say, $F$ are defined as follows: for $(\delta \mu, \delta \eta, \delta s) \epsilon$ $T_{(\mu, \eta, s)} \varsigma^{*} \approx \varsigma^{*},(\delta F / \delta \mu, \delta F / \delta \eta, \delta F / \delta s)$ is the element of s such that

$$
\left\langle(\delta \mu, \delta \eta, \delta s),\left(\frac{\delta F}{\delta \mu}, \frac{\delta F}{\delta \eta}, \frac{\delta F}{\delta s}\right)\right\rangle=\left(D_{\mu} F\right) \delta \mu+\left(D_{\eta} F\right) \delta \eta+\left(D_{s} F\right) \delta s .
$$

We shall find that with the push-forward action of ${ }^{(3)} \mathscr{D}$ on $\mathscr{F}$ and $\mathscr{F}^{*}$, the left Lie bracket on $\mathscr{X}$, and the specific evolution equations at hand (see Sect. 3 ), \{\}$_{+}$ is the Lie-Poisson structure we want. This is consistent with the conclusions for a non-relativistic perfect fluid, as discussed in Marsden et al. (1984a). Integration by parts shows that

$$
\begin{aligned}
\{F, H\}_{+}(\mu, \eta, s)=\langle & \left(-\mathscr{L}_{(\delta \boldsymbol{H} / \delta \mu)} \mu-\eta d \frac{\delta H}{\delta \eta}+\frac{\delta H}{\delta s} d s,-\mathscr{L}_{(\delta \boldsymbol{H} / \delta \mu)} \eta,-\mathscr{L}_{(\delta \boldsymbol{H} / \delta \mu)} s\right), \\
& \left.\left(\frac{\delta F}{\delta \mu}, \frac{\delta F}{\delta \eta}, \frac{\delta F}{\delta s}\right)\right\rangle .
\end{aligned}
$$


Note that since $(\delta H / \delta s) \in \mathscr{F} *$ is a density,

$$
\begin{aligned}
\left\langle s, \rho^{\prime}\left(\frac{\delta F}{\delta \mu}\right) \cdot \frac{\delta H}{\delta s}\right\rangle & =\left\langle s,-\mathscr{L}_{(\delta F / \delta \mu)} \frac{\delta H}{\delta s}\right\rangle=\left\langle\mathscr{L}_{(\delta F / \delta \mu)} s, \frac{\delta H}{\delta s}\right\rangle=\left\langle d s\left(\frac{\delta F}{\delta \mu}\right), \frac{\delta H}{\delta s}\right\rangle \\
& =\left\langle\frac{\delta H}{\delta s} d s, \frac{\delta F}{\delta \mu}\right\rangle .
\end{aligned}
$$

On the other hand, since $(\delta H / \delta \eta) \in \mathscr{F}$ is a scalar, $\left\langle\eta, \rho^{\prime}(\delta F / \delta \mu) \cdot \delta H / \delta \eta\right\rangle=$ $\left\langle\eta, \quad\left(-\mathscr{L}_{\delta F / \delta \mu}\right)(\delta H / \delta \eta)\right\rangle=\langle\eta, \quad-d(\delta H / \delta \eta)(\delta F / \delta \mu)\rangle=\langle-\eta d(\delta H / \delta \eta), \quad \delta F / \delta \mu\rangle$. This explains the difference in signs in front of the $\eta d(\delta H / \delta \eta)$ and $(\delta H / \delta s) d s$ terms.

We remark that if the pull-back action of ${ }^{(3)} \mathscr{D}$ on $\mathscr{F}$ and $\mathscr{F} *$ (i.e. $\rho(\sigma) \cdot f=f \circ \sigma$, $\rho(\sigma) \cdot(f \sqrt{g})=(f \circ \sigma)(\operatorname{det} D \sigma) \sqrt{g})$ had been chosen, if the Lie bracket on $\mathscr{X}$ were the "ordinary one" (i.e. $[X, Y]_{R}=\mathscr{L}_{X} Y$ ), then given the same fluid evolution equations,

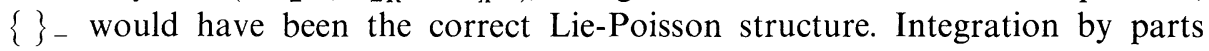
shows that in such a case, $\{F, H\}_{-}$is indeed equal to the right-hand-side of (1C.15).

Let $H$ be a function on $\left(\sigma^{*},\{\}_{+}\right)$. The Hamiltonian vector field $X_{H}$ is a vector field on $\varsigma^{*}$ such that for all functions $F$ on $\varsigma^{*}$,

$$
(D F)_{\mid(\mu, \eta, s)}\left(X_{H}\right)=\{F, H\}_{+}(\mu, \eta, s) .
$$

Comparing (1C.16) with (1C.15), we see that

$$
X_{H}(\mu, \eta, s)=\left(-\mathscr{L}_{(\delta H / \delta \mu)} \mu-\eta d \frac{\delta H}{\delta \eta}+\frac{\delta H}{\delta s} d s,-\mathscr{L}_{(\delta H / \delta \mu)} \eta,-\mathscr{L}_{(\delta \boldsymbol{H} / \delta \mu)} s\right)
$$

Note also that, by virtue of (1C.10, 1C.12), we have

$$
X_{H}(\mu, \eta, s)=-\left[\operatorname{ad}\left(\frac{\delta H}{\delta \mu}, \frac{\delta H}{\delta \eta}, \frac{\delta H}{\delta s}\right)\right]^{*}(\mu, \eta, s)=\left(\frac{\delta H}{\delta \mu}, \frac{\delta H}{\delta \eta}, \frac{\delta H}{\delta s}\right)_{\delta^{*}}(\mu, \eta, s),
$$

So $X_{H}$ is tangent to coadjoint orbits.

On any manifold $P$ with a Poisson structure $\{$,$\} (a derivation on the function$ in each slot, real bilinear, antisymmetric and obeying the Jacobi identity), it is a direct consequence of the chain rule and the definition of Hamiltonian vector fields that if $c: \mathbb{R} \rightarrow P$ is a curve, then

$$
\frac{d}{d t} F(c(t))=\{F, H\}(c(t)) \text { for all functions } F \text { iff } \frac{d}{d t} c(t)=X_{H}(c(t)) .
$$

In the case of $\left(s^{*},\{\}_{+}\right)$, the above observation, together with (1C.17), gives

Proposition 1. Let $H$ be a Hamiltonian on $\left(s^{*},\{\}_{+}\right)$, and let $(\mu, \eta, s)(t)$ be a curve in $s^{*}$. The following statements are equivalent:

(i) $\partial_{t}(\mu, \eta, s)=\left(-\mathscr{L}_{(\delta H / \delta \mu)} \mu-\eta d \frac{\delta H}{\delta \eta}+\frac{\delta H}{\delta s} d s,-\mathscr{L}_{(\delta H / \delta \mu)} \eta,-\mathscr{L}_{(\delta H / \delta \mu)} s\right)$. 
(ii) $\partial_{t} F((\mu, \eta, s)(t))=\{F, H\}_{+}((\mu, \eta, s)(t))$ for all functions $F$ on $s^{*}$ (these are known as the Lie-Poisson equations on $\left.\left(\varsigma^{*},\{\}_{+}\right)\right)$.

(iii) $(\mu, \eta, s)(t)$ is an integral curve of $X_{H}$.

\section{The Field Equations of the Einstein-Euler System and Their} $(3+1)$ Decomposition

Assuming that there is no heat exchange between adjacent fluid elements, the stressenergy tensor for our relativistic fluid is

$$
T=e u \otimes u+\left({ }^{(4)} g+u \otimes u\right) p,
$$

(Misner et al., 1972; Ex. 22.7). Equivalently, by (1A.3) and (1B.2),

$$
T^{\alpha \beta}=n h^{3} J^{\alpha} J^{\beta}+p g^{\alpha \beta} .
$$

In geometrical units with $8 \pi G=c=1$, the field equations are

and

$$
\begin{aligned}
\operatorname{Ein}_{\alpha \beta} & =T_{\alpha \beta}, \\
T_{; \beta}^{\alpha \beta} & \left.=0 \quad \text { (i.e. }{ }^{(4)} \nabla \cdot T=0\right),
\end{aligned}
$$

$$
\left.s_{, \alpha} u^{\alpha}=0 \quad \text { (i.e. }{ }^{(4)} \nabla_{u} s=0\right) .
$$

Equation (2.5) is the statement that entropy is conserved along flow lines. This is equivalent to rest space energy conservation (the first law of thermodynamics) and the assumption of no heat exchange. We are therefore dealing with an adiabatic flow with no shock waves. (How the present formulation should deal with shocks is not yet clear.)

If the basic variables were taken to be the metric ${ }^{(4)} g$, the fluid's world lines, and $s$, and if an equation of state of the form $e=e\left({ }^{(4)} g+u \otimes u, s\right)$ were chosen, then as in Carter (1973), the field Eqs. $(2.3,2.4)$ would result from the variation of the action $\int d^{4} x \sqrt{-g}\left(\frac{1}{2}{ }^{(4)} R-e\right)$. This action is formally the same as one used by Taub (1954), in which case the equation of state was $e=e(n, s)$.

In our case, the field Eq. (2.3) is derivable from the following variational principle. If we vary the action $\int d^{4} x \sqrt{-g}\left(\frac{1}{2}^{(4)} R+p(h, s)\right)$ with respect to ${ }^{(4)} g_{\alpha \beta}$ and set the coefficient of $\delta g_{\alpha \beta}$ equal to zero, we get

$$
\operatorname{Ein}^{\alpha \beta}=2 \sqrt{-g}-1 \frac{\delta(p(h, s) \sqrt{-g})}{\delta g_{\alpha \beta}} .
$$

Using $n=\partial_{h} p$ and $-h^{-2}=J^{\alpha} J^{\beta} g_{\alpha \beta}$, the right-hand-side of (2.6) is readily computed to be $T^{\alpha \beta}$ as given in (2.2).

We now $(3+1)$ decompose the Einstein field equation. Taking the "I, ," projection of $\sqrt{-g} \operatorname{Ein}^{\alpha \beta}=\sqrt{-g} T^{\alpha \beta}$, we get the evolution equations

$$
\partial_{t} g_{i j}=2 N \sqrt{g}^{-1}\left(\pi_{i j}-\frac{1}{2} g_{i j} \operatorname{tr} \pi\right)+\mathscr{L}_{M} g_{i j},
$$

and

$$
\begin{aligned}
\partial_{t} \pi^{i j}= & N\left[S_{g}(\pi, \pi)-\sqrt{g} \operatorname{Ein}\left({ }^{(3)} g\right)\right]^{i j}+\sqrt{g}\left(\operatorname{Hess}^{i j}+g^{i j} \Delta\right) \cdot N+\mathscr{L}_{M} \pi^{i j} \\
& +N \sqrt{g}\left(n h^{3} J_{\|}^{i} J_{\|}^{j}+p g^{i j}\right) .
\end{aligned}
$$


Here, $\operatorname{Hess}^{i j} \cdot N=N^{\mid l j}, \Delta N=-N_{\mid l}^{k_{1}}$, and

$$
\left[S_{g}(\pi, \pi)\right]^{i j}=-2 \sqrt{g^{-1}}\left(\pi^{i k} \pi_{k}^{j}-\frac{1}{2} \pi^{i j} \operatorname{tr} \pi\right)+\frac{1}{2} \sqrt{g}^{-1} g^{i j}\left(\pi^{k l} \pi_{k l}-\frac{1}{2}(\operatorname{tr} \pi)^{2}\right)
$$

is the spray of the DeWitt metric (Fischer and Marsden, 1979). Equation (2.7) may be viewed as the defining relation for the conjugate momenta $\pi^{i j}$. We also remind the reader that we write $\sqrt{g}=\left(\operatorname{det} g_{i j}\right)^{1 / 2}$ and $\sqrt{-g}=\left(-\operatorname{det} g_{\alpha \beta}\right)^{1 / 2}=N \sqrt{g}$.

To simplify later discussions, we introduce the vacuum superhamiltonian

$$
\mathscr{H}_{\mathrm{vac}}=\sqrt{g}^{-1}\left[\pi^{k l} \pi_{k l}-\frac{1}{2}(\operatorname{tr} \pi)^{2}\right]-\sqrt{g} R,
$$

and vacuum supermomentum

$$
\mathscr{J}_{v a c}=-2 \nabla \cdot \pi
$$

as well as the following adjoint notation, following Fischer and Marsden (1979). If $\mathscr{K}=\mathscr{K}(g, \pi)$ is a scalar density, we define the $L^{2}$-adjoints $\left(D_{g} \mathscr{K}\right)^{*}$ and $\left(D_{\pi} \mathscr{K}\right)^{*}$ via integration by parts:

$$
\int_{\Sigma}\left\langle\left(D_{g} \mathscr{K}\right)^{*} \cdot 1, \delta g\right\rangle+\left\langle\left(D_{\pi} \mathscr{K}\right)^{*} \cdot 1, \delta \pi\right\rangle=\int_{\Sigma}\left(D_{g} \mathscr{K}\right) \delta g+\left(D_{\pi} \mathscr{K}\right) \delta \pi
$$

The evolution Eqs. $(2.7,2.8)$ can be compactly rewritten in the following adjoint form:

$$
\begin{gathered}
\partial_{t} g=\left(D_{\pi} \mathscr{H}_{\mathrm{vac}}\right)^{*} \cdot N+\left(D_{\pi} \mathscr{J}_{\mathrm{vac}}\right)^{*} \cdot M \\
\partial_{t} \pi=-\left(D_{g} \mathscr{H}_{\mathrm{vac}}\right)^{*} \cdot N-\left(D_{g} \mathscr{J}_{\mathrm{vac}}\right)^{*} \cdot M+N\left(n h^{3} J_{\|} \otimes J_{\|}+p g^{\#}\right) \sqrt{g}
\end{gathered}
$$

As usual, the " $\perp, \perp$ ” and “ $\perp$, $\|$ ” projections of (2.3) yield the constraints. More precisely, one obtaines

where

$$
\begin{gathered}
\mathscr{H}\left(g, \pi, J^{\perp}, J_{\|}, s\right)=0, \\
\mathscr{J}\left(g, \pi, J^{\perp}, J_{\|}, s\right)=0,
\end{gathered}
$$

$\mathscr{H}=-2\left(“ \perp, \perp ”\right.$ projection of $\left.\operatorname{Ein}_{\alpha \beta}-T_{\alpha \beta}\right) \sqrt{g}=\mathscr{H}_{\text {vac }}+2 n h^{3}\left(J^{\perp}\right)^{2} \sqrt{g}-2 p \sqrt{g}$,

and

$$
\mathscr{J}=-2\left(“ \perp, \| ” \text { projection of } \operatorname{Ein}_{\alpha \beta}-T_{\alpha \beta}\right) \sqrt{g}=\mathscr{J}_{\text {vac }}-2 n h^{3} J^{\perp} J_{\|}^{b} \sqrt{g} .
$$

Next, we $(3+1)$ decompose the conservation law ${ }^{(4)} \nabla \cdot T=0$. The projection of ${ }^{(4)} \nabla \cdot T=0$ along $u$, that is $u_{\alpha}\left(T^{\beta \alpha}\right)_{; \beta}=0$, and use of the entropy Eq. (2.5), gives the continuity equation

$$
\left(n h J^{\beta}\right)_{; \beta}=0
$$

(equivalently, $\left(n u^{\beta}\right)_{; \beta}=0$ ) or, using the torsion-freeness of our connection,

$$
\left(n h J^{\beta} \sqrt{-g}\right)_{, \beta}=0 .
$$

A routine computation reexpresses (2.19) as

$$
\partial_{t}\left(n h J^{\perp} \sqrt{g}\right)=-\mathscr{L}_{\left(-M+N J_{\|}\left(J^{\perp}\right)^{-1}\right)}\left(n h J^{\perp} \sqrt{g}\right) .
$$


The projection of ${ }^{(4)} \nabla \cdot T=0$ orthogonal to $u$ is

$$
\left(g_{\xi \alpha}+u_{\xi} u_{\alpha}\right)\left(\left(n h u^{\beta} u^{\xi}\right)_{; \beta}+p^{, \xi}\right)=0 .
$$

Let us digress a little; (2.21) can be rewritten as

$$
(p+e)^{(4)} \nabla_{u} u=-P \cdot\left({ }^{(4)} \nabla p\right)
$$

where

$$
P={ }^{(4)} g+u \otimes u
$$

is the projection tensor onto the 3-dimensional subspace orthogonal to $u$. Equation (2.22) is the relativistic analogue of the non-relativistic Euler equation-see Misner et al. (1973), pp. 159 and 565. We also see that the quantity $p+e$ (hence $n h$ ) plays the role of a mass - the "inertial mass" (per unit rest space 3-volume). Let us return to (2.21). Using the continuity Eq. (2.18), the entropy Eq. (2.5), and the relation $J=u h^{-1},(2.21)$ can be rewritten as

$$
\left(n h^{3} J^{\beta} J_{\alpha}\right)_{; \beta}=-n h_{, \alpha}+n T s_{, \alpha},
$$

or equivalently,

$$
\left(\sqrt{-g} n h^{3} J^{\beta} J_{\alpha}\right)_{; \beta}=-\sqrt{-g} n h_{, \alpha}+\sqrt{-g} n T s_{, \alpha} .
$$

Note that if $A$ is a tensor, the covariant derivative of the tensor density $A \sqrt{-g}$ is defined as

$$
(A \sqrt{-g})_{; \beta}=(A \sqrt{-g})_{, \beta}+\text { corrections as if }(A \sqrt{-g}) \text { were a tensor }-(A \sqrt{-g})\left\{{ }^{v}{ }_{\nu \beta}\right\} \text {. }
$$

Now write out the covariant derivative in (2.25), set $\alpha=i$, and after a lengthy computation, one gets

$$
\begin{aligned}
\partial_{t}\left(n h^{3} J_{\|}^{b} J^{\perp} \sqrt{g}\right)= & -\mathscr{L}_{\left(-M+N J_{\|}\left(J^{\perp}\right)^{-1}\right)}\left(n h^{3} J_{\|}^{b} J^{\perp} \sqrt{g}\right) \\
& -\left(n h J^{\perp} \sqrt{g}\right) d\left(N\left(J^{\perp}\right)^{-1}\right)+(n N T \sqrt{g}) d s .
\end{aligned}
$$

Here, $d$ denotes the exterior derivative on $\Sigma$. (For example, $d s=s_{, i} d x^{i}$, rather than $\left.s_{, \alpha} d x^{\alpha}\right)$.

Finally, the $(3+1)$ decomposition of the entropy Eq. (2.5) gives

$$
\partial_{t} s=-\mathscr{L}_{\left(-M+N J_{\|}\left(J^{\perp}\right)-1\right)} S .
$$

The physical meaning of the quantities $n h J^{\perp} \sqrt{g}$ and $n h^{3} J_{\|}^{b} J^{\perp} \sqrt{g}$ is given in Sect. 3 .

Let us examine the evolution Eqs. $(2.12,2.13,2.20,2.26,2.27)$ and the constraint Eqs. $(2.14,2.15)$. The constraints are statements about $\left(g, \pi, J^{\perp}, J_{\mid}, s:=\left.s\right|_{\Sigma}\right)$, which are fields on $\Sigma$ obtained by the usual $(3+1)$ decomposition of the spacetime fields $\left({ }^{(4)} g,{ }^{(4)} J, s\right)$. However, the evolution Eqs. $(2.20,2.26)$ are for the quantities $n h J^{\perp} \sqrt{g}$ and $n h^{3} J_{\|}^{b} J^{\perp} \sqrt{g}$ rather than for $J^{\perp}$ and $J_{\|}$directly. Nevertheless, the evolution equations for $J^{\perp}$ and $J_{\|}$can be written down, in principle. The reason is that the map $\left(J^{\perp}, J_{\|}\right) \mapsto\left(n h J^{\perp} \sqrt{g}, n h^{3} J_{\|}^{\mathrm{b}} J^{\perp} \sqrt{g}\right)$ is invertible for fixed $s$ and $g$ (see Sect. 3), so in principle, $J^{\perp}$ and $J_{\|}$are $(s, g)$-dependent functionals of 
$\left(n h J^{\perp} \sqrt{g}, n h^{3} J_{\|}^{\natural} J^{\perp} \sqrt{g}\right)$, that is,

$$
\left(J^{\perp}, J_{\|}\right)=\left(J^{\perp}, J_{\|}\right)\left(n h J^{\perp} \sqrt{g}, n h^{3} J_{\|}^{b} J^{\perp} \sqrt{g}, s, g\right) .
$$

Using the chain rule and the evolution Eqs. $(2.20,2.26,2.12,2.27)$, we can obtain evolution equations for $\left(J^{\perp}, J_{\|}\right)$. Unfortunately, for a general equation of state, an explicit formula for the functional dependence in (2.28) is at best difficult to write down. Also, there is no good reason for us to expect that the resulting evolution equations for $\left(J^{\perp}, J_{\|}\right)$are any simpler than those for $\left(n h J^{\perp} \sqrt{g}, n h^{3} J_{\|}^{\text {b }} J^{\perp} \sqrt{g}\right)$. Due to these aforementioned considerations, we shall not regard $\left(J^{\perp}, J_{\|}\right)$as the basic fluid variables on $\Sigma$. It will be shown in Sect. 3 that a constant multiple of the set $\left(n h J^{\perp} \sqrt{g}, n h^{3} J_{\|}^{b} J^{\perp} \sqrt{g}\right)$ is a useful choice of basic fluid variables on $\Sigma$.

\section{A Hamiltonian Description of the Dynamical Einstein-Euler System}

Define the fluid variables

$$
\begin{aligned}
& \mu:=2 n h^{3} J_{\|}^{\natural} J^{\perp} \sqrt{g}, \\
& \pi:=2 n h J^{\perp} \sqrt{g} .
\end{aligned}
$$

We have $(\mu, \eta) \in \mathscr{X}^{*} \times \mathscr{F}^{*}$. The factor of 2 present in these definitions is essential for our purposes. We will pinpoint its origin later in this section.

Let us first give the physical interpretation of $n h J^{\perp} \sqrt{g}$ and $n h^{3} J_{\|}^{\text {b }} J^{\perp} \sqrt{g}$ or, equivalently, $n u^{\perp} \sqrt{g}$ and $n h u_{\|}^{b} u^{\perp} \sqrt{g}$. As described before, $n$ and $n h$ are, respectively, the fluid's particle number and inertial mass (per unit 3-volume in the fluid's rest space). The subspace orthogonal to $u$ is the fluid's rest space, whereas that orthogonal to the unit normal $n$ is the tangent space on a hypersurface; $u^{\perp}=u_{\perp}=-{ }^{(4)} g(u, n)$ is the conversion factor between 3-volume measurements in the fluid's rest space and those done on the hypersurface; as a consistency check, note that if the fluid is at rest in the hypersurface (i.e. $u=N^{-1} \partial_{t}$ ), we have $u^{\perp}=1$. $\sqrt{g}$ is, of course, the 3 -volume of a coordinate cube on the hypersurface. Thus, $n u^{\perp} \sqrt{g}$ and $n h u_{\|}^{b} u^{\perp} \sqrt{g}$ are, respectively, the number of baryons and the amount of inertial momentum contained in each coordinate cube. We remark that in the gauge $\partial_{t}=J, \eta$ reduces to Taub's conserved scalar density while $\mu$ corresponds to his conserved spatial one-form (in the case of isentropic flows). See Taub (1969).

Next, let us show that $\left(J^{\perp}, J_{\|}\right)$are $(s, g)$-dependent functionals of $(\mu, \eta)$, that is,

$$
\left(J^{\perp}, J_{\|}\right)=\left(J^{\perp}, J_{\|}\right)(\mu, \eta, s, g),
$$

provided that assumptions (1A.4) (non-zero intertial mass) and (1A.11) (speed of sound in fluid not exceeding the speed of light in vacuum) are valid. The functional dependence (3.3) is obtainable through an application of the inverse function theorem, as follows. For each choice of fixed $s$ and $g$, one can check that the Jacobian matrix of the map

$$
\left(J^{\perp}, J_{\|}\right) \stackrel{\Omega}{\rightarrow}\left(2 \mathrm{nh} J^{\perp} \sqrt{g}, 2 n h^{3} J_{\|}^{\mathrm{b}} J^{\perp} \sqrt{g}\right)
$$


is the 4-by-4 matrix

$$
D \Omega=\left(\begin{array}{ll}
a_{00} & a_{0 j} \\
a_{i 0} & a_{i j}
\end{array}\right), \quad 1 \leqq i, j \leqq 3,
$$

where

$$
\begin{aligned}
a_{00} & =2 \sqrt{g} n h\left[1-\left(h J^{\perp}\right)^{2}\left(1+v^{-2}\right)\right], \\
a_{0 j} & =2 \sqrt{g} n h\left(h J^{\perp}\right)\left(h J_{\|_{j}}\right)\left(1+v^{-2}\right), \\
a_{i 0} & =2 \sqrt{g} n h^{2}\left(h J_{\|_{i}}\right)\left[1-\left(h J^{\perp}\right)^{2}\left(1+v^{-2}\right)-2\left(h J^{\perp}\right)^{2}\right], \\
a_{i j} & =2 \sqrt{g} n h^{2}\left(h J^{\perp}\right)\left[g_{i j}+\left(h J_{\|_{2}}\right)\left(h J_{\|_{j}}\right)\left(1+v^{-2}\right)+2\left(h J_{\|_{2}}\right)\left(h J_{\|_{J}}\right)\right] .
\end{aligned}
$$

Recall that $v$ is the speed of sound in the fluid, as defined in (1A.10). We perform the following three elementary row/column operations on $D \Omega$ which leave $\operatorname{det}(D \Omega)$ unchanged: Subtract $h^{2} J_{\| i}$ times row zero from row $i$, then add $\left(h J^{\perp}\right)^{-1}\left(h J_{\| j}\right)$ times column zero to column $j$, then subtract $\left(\left(h J^{\perp}\right)^{2} h\right)^{-1}\left(h J_{\|}^{i}\right)$ times row $i$ (summed on $i)$ from row zero and use the identity $\left(1\right.$ B.8) in the form $h^{2}\left(J_{\|}\right)^{2}=\left(h J^{\perp}\right)^{2}-1$. These elementary operations reduce the matrix $D \Omega$ to

$$
\left(\begin{array}{cc}
2 \sqrt{g} n h\left[h^{2}\left(J_{\|}\right)^{2}-\left(h J^{\perp}\right)^{2} v^{-2}\right] & 0 \\
2 \sqrt{g} n h^{2}\left(h J_{\|_{2}}\right)\left[-2\left(h J^{\perp}\right)^{2}\right] & 2 \sqrt{g} n h^{2}\left(h J^{\perp}\right) g_{i j}
\end{array}\right),
$$

hence,

$$
\begin{aligned}
\operatorname{det}(D \Omega) & =2 \sqrt{g} n h\left(h J^{\perp}\right)^{2}\left[\left(J_{\|}\right)^{2}\left(J^{\perp}\right)^{-2}-v^{-2}\right] \operatorname{det}\left(2 \sqrt{g} n h^{2}\left(h J^{\perp}\right) g_{i j}\right) \\
& =(2 n h)^{4}(\sqrt{g})^{6} h^{3}\left(h J^{\perp}\right)^{5}\left[\left(J_{\|}\right)^{2}\left(J^{\perp}\right)^{-2}-v^{-2}\right],
\end{aligned}
$$

which is non-zero since $n h \neq 0(1 \mathrm{~A} .4), J^{\perp} \neq 0(1 \mathrm{~B} .10),\left(J_{\|}\right)^{2}\left(J^{\perp}\right)^{-2}<1$ (1B.11) and $v^{-2} \geqq 1$ (1A.11). The functional dependence in (3.3) then follows by the inverse function theorem.

For the rest of this section, unless otherwise stated, all dependences on $\left(J^{\perp}, J_{\|}\right)$, whether explicit or implicit, are to be understood as dependences on $(\mu, \eta, s, g)$ through Eq. (3.3).

We take the phase space of the $(3+1)$ decomposed Einstein-Euler system to be $T^{*} \mathscr{M} \times \jmath^{*}$, where $\mathscr{M}$ is the manifold of all Riemannian metrics on $\Sigma$ and $s^{*}$ is the semi-direct product $\mathscr{X}^{*} \propto\left(\mathscr{F}^{*} \times \mathscr{F}\right)$ (see Sect. 1C). The basic fields on $\Sigma$ are $(g, \pi, \mu, \eta, s)$, the atlas fields (Isenberg, 1980) are $N$ and $M$, and the evolution equations are

$$
\begin{aligned}
& \partial_{t} g=\left(D_{\pi} \mathscr{H}_{\mathrm{vac}}\right)^{*} \cdot N+\left(D_{\pi} \mathscr{J}_{\mathrm{vac}}\right)^{*} \cdot M \\
& \partial_{t} \pi=-\left(D_{g^{2}} \mathscr{H}_{\mathrm{vac}}\right)^{*} \cdot N-\left(D_{g} \mathscr{J}_{\mathrm{vac}}\right)^{*} \cdot M+N\left(n h^{3} J_{\|} \otimes J_{\|}+p g^{\#}\right) \sqrt{g} \\
& \partial_{t} \mu=-\mathscr{L}_{\left(-M+N J_{\|}\left(J^{\perp}\right)^{-1}\right)} \mu-\eta d\left(N\left(J^{\perp}\right)^{-1}\right)+(2 n N T \sqrt{g}) d s \\
& \partial_{t} \eta=-\mathscr{L}_{\left(-M+N J_{\|}\left(J^{\perp}\right)^{-1}\right)} \eta \\
& \partial_{t} s=-\mathscr{L}_{\left(-M+N J_{\|}\left(J^{\perp}\right)^{-1}\right)} S
\end{aligned}
$$


with the following constraints on the initial data

$$
\begin{gathered}
\mathscr{H}(g, \pi, \mu, \eta, s)=\mathscr{H}_{\mathrm{vac}}+\left(J_{\|}^{i} \mu_{i}+\eta\right)\left(J^{\perp}\right)^{-1}-2 p \sqrt{g}=0, \\
\mathscr{J}(g, \pi, \mu)=\mathscr{J}_{\mathrm{vac}}-\mu=0 .
\end{gathered}
$$

Equations (3.4) $\rightarrow(3.10)$ are merely rewrites of $(2.12,2.13,2.26,2.20,2.27)$ and $(2.14$, $2.15)$, with the use of the identity $2 n h^{3}\left(J^{\perp}\right)^{2} \sqrt{g}=\left(J_{\|}^{i} \mu_{i}+\eta\right)\left(J^{\perp}\right)^{-1}$. The motivation for using such an identity to rewrite $\mathscr{H}$ will become clear. Note also that $(3.6,3.7)$ are obtained by multiplying $(2.26,2.20)$ by a factor of 2 .

Define the Hamiltonian $H=H(g, \pi, \mu, \eta, s, N, M)$ by

$$
H=\int_{\Sigma}\left(N \mathscr{H}+M^{i} \mathscr{J}_{i}\right)
$$

This is the usual Dirac/canonical Hamiltonian (Dirac, 1964; Hansen et al., 1976). Note that the fluid part of $\mathscr{H}$ is $2 n h^{3}\left(J^{\perp}\right)^{2} \sqrt{g}-2 p \sqrt{g}=2 n h\left(1+\left(u_{\|}\right)^{2}\right) \sqrt{g}-$ $2 p \sqrt{g}=2\left(u^{\perp}\right)^{-1}\left(e u^{\perp} \sqrt{g}+n h\left(u_{\|}\right)^{2} u^{\perp} \sqrt{g}\right)$. The term $e u^{\perp} \sqrt{g}$ is the rest energy in each coordinate cube on $\Sigma$, while $n h\left(u_{\|}\right)^{2} u^{\perp} \sqrt{g}$ is essentially the amount of inertial kinetic energy in each such coordinate cube.

The aforementioned fluid part of $\mathscr{H}$ may also be rewritten as $\sqrt{\eta^{2} h^{2}+g^{i j} \mu_{i} \mu_{j}}-$ $2 p \sqrt{g}$, through the use of the identity $2 n h^{3}\left(J^{\perp}\right)^{2} \sqrt{g}=\left(J_{\|}^{i} \mu_{i}+\eta\right)\left(J^{\perp}\right)^{-1}$, as well as Eqs. (3.1), (3.2), and (1B.8). Such is essentially the Hamiltonian density derived by Holm and Kupershmidt (1984) for special-relativistic (that is, relativistic but nongravitating) perfect fluids. It is our impression that less algebra is involved in the derivations of this paper using our form of the fluid Hamiltonian rather than the numerically identical Holm-Kupershmidt form.

We are now ready to show that $H$ generates the evolution of $(g, \pi, \mu, \eta, s)$ in $T^{*} \mathscr{M} \times \varsigma^{*}$. First let us consider $H$ as a function on $\diamond^{*}$. By Proposition 1 (Sect. 1C), one sees that the evolution Eqs. (3.6), (3.7) and (3.8) define integral curves of $H$ (and hence are equivalent to the Lie-Poisson equation of $H$ on $\varsigma^{*}$ ) provided that $H$ satisfies

$$
\begin{aligned}
& \frac{\delta H}{\delta \mu}=-M+N J_{\|}\left(J^{\perp}\right)^{-1}, \\
& \frac{\delta H}{\delta \eta}=N\left(J^{\perp}\right)^{-1},
\end{aligned}
$$

and

$$
\frac{\delta H}{\delta s}=2 n N T \sqrt{g}
$$

To facilitate the verification (3.12), (3.13) and (3.14), we shall use implicit differentiation. The expression for $\mathscr{H}$, namely $\mathscr{H}_{\text {vac }}+\left(J_{\|}^{i} \mu_{i}+\eta\right)\left(J^{\perp}\right)^{-1}-$ $2 p\left(h\left(J^{\perp}, J_{\|}, g\right), s\right) \sqrt{g}$, can be regarded as a functional $\mathscr{H}^{\prime}\left(g, \pi, \mu, \eta, s, J^{\perp}, J_{\|}\right)$ which is numerically equal to $\mathscr{H}(g, \pi, \mu, \eta, s)$ through the use of the implicit 
dependence (3.3) of $\left(J^{\perp}, J_{\|}\right)$on $(\mu, \eta, s, g)$. The statements

$$
\begin{aligned}
& \partial_{J^{\perp}} \mathscr{H}^{\prime}=-\left(J^{\perp}\right)^{-2}\left(J_{\|}^{i} \mu_{i}+\eta\right)+2 \sqrt{g} n h^{3} J^{\perp}=0, \\
& \partial_{J_{\|}^{i}} \mathscr{H}^{\prime}=\mu_{i}\left(J^{\perp}\right)^{-1}-2 \sqrt{g} n h^{3} J_{\|_{\imath}}=0,
\end{aligned}
$$

are easily verified using $d p=n d h-n T d s$ and

$$
\begin{aligned}
& \partial_{J^{\perp}} h=-J^{\perp} h^{3} \\
& \partial_{J_{\|}} h=J_{\|_{\imath}} h^{3},
\end{aligned}
$$

as well as the defining relations $(3.1,3.2)$, which have become identities in view of (3.3).

Using $(3.15,3.16)$, we have, for any $\omega \in \mathscr{X}^{*}$,

$$
\begin{aligned}
\left\langle\omega, \frac{\delta H}{\delta \mu}\right\rangle & =\left(D_{\mu} H\right) \cdot \omega \\
& =\int_{\Sigma} N\left(D_{\mu_{\imath}} \mathscr{H}\right) \omega_{i}+M^{k}\left(D_{\mu_{\imath}} \mathscr{J}_{k}\right) \omega_{i} \\
& =\int_{\Sigma} N\left(J^{\perp}\right)^{-1} J_{\|}^{i} \omega_{i}+N\left[\left(\partial_{J \perp} \mathscr{H}^{\prime}\right) \cdot D_{\mu_{i}} J^{\perp}+\left(\partial_{J_{\|}^{j}} \mathscr{H}^{\prime}\right) \cdot D_{\mu_{l}} J_{\|}^{j}\right] \omega_{i}-M^{i} \omega_{i} \\
& =\left\langle\omega,-M+N J_{\|}\left(J^{\perp}\right)^{-1}\right\rangle .
\end{aligned}
$$

This verifies $(3.12)$, and $(3.13,3.14)$ are similar. Hence our Hamiltonian $H$ does generate the evolution of $(\mu, \eta, s)$.

We next turn to the evolution equations for $(g, \pi)$. Let us regard the Hamiltonian $H$ as a function on $T^{*} \mathscr{M}$. Being a cotangent bundle, $T^{*} \mathscr{M}$ has a canonical symplectic structure, with associated Poisson bracket

$$
\left\{F_{1}, F_{2}\right\}(g, \pi)=\left\langle\frac{\delta F_{1}}{\delta g}, \frac{\delta F_{2}}{\delta \pi}\right\rangle-\left\langle\frac{\delta F_{2}}{\delta g}, \frac{\delta F_{1}}{\delta \pi}\right\rangle .
$$

For $F_{i}(g, \pi)=\int_{\Sigma} \mathscr{F}_{i}(g, \pi)$, the bracket becomes

$$
\left\{F_{1}, F_{2}\right\}(g, \pi)=\int_{\Sigma}\left\langle\left(D_{g} \mathscr{F}_{1}\right)^{*} \cdot 1,\left(D_{\pi} \mathscr{F}_{2}\right)^{*} \cdot 1\right\rangle-\left\langle\left(D_{\pi} \mathscr{F}_{1}\right)^{*} \cdot 1,\left(D_{g} \mathscr{F}_{2}\right)^{*} \cdot 1\right\rangle
$$

(as in Fischer and Marsden 1979), where the adjoints are defined as in (2.11). For our Hamiltonian $H=\int_{\Sigma}(N \mathscr{H}+M \mathscr{J})$, the Hamiltonian vector field $X_{H}$ (defined by $\left.(\mathrm{DF}) \cdot X_{H}=\{F, H\}\right)$ is, for the $(g, \pi)$ variables (holding $\mu, \eta$ and $s$ constant):

$$
X_{H}(g, \pi)=\left(\begin{array}{cc}
0 & 1_{6 \times 6} \\
-1_{6 \times 6} & 0
\end{array}\right)\left(\begin{array}{c}
\left(D_{g} \mathscr{H}\right)^{*} \cdot N+\left(D_{g} \mathscr{J}\right)^{*} \cdot M \\
\left(D_{\pi} \mathscr{H}\right)^{*} \cdot N+\left(D_{\pi} \mathscr{J}\right)^{*} \cdot M
\end{array}\right)
$$

As usual, the symplectic matrix $\left(\begin{array}{cc}0 & 1 \\ -1 & 0\end{array}\right)$ converts elements of $T_{(g, \pi)}^{*}\left(T^{*} \mathscr{M}\right)$ into those of $T_{(g, \pi)}\left(T^{*} \mathscr{M}\right)$. (The form (3.20) is equivalent to the adjoint form of Fischer and Marsden, 1979.)

Using $d p=n d h-n T d s$, as well as

$$
\partial_{g_{\imath j}} h=\frac{1}{2} h^{3} J_{\|}^{i} J_{\|}^{j}
$$


and

$$
\partial_{g_{i},} \sqrt{g}=\frac{1}{2} \sqrt{g} g^{i j}
$$

we have

$$
D_{g} \mathscr{H}=D_{g} \mathscr{H}_{\mathrm{vac}}-n h^{3} J_{\|} \otimes J_{\|} \sqrt{g}-p \sqrt{g} g^{\#}+\left(\partial_{J \perp} \mathscr{H}^{\prime}\right) D_{g} J^{\perp}+\left(\partial_{J_{\|}} \mathscr{H}^{\prime}\right) D_{g} J_{\|}
$$

which, by (3.15), (3.16), becomes $D_{g} \mathscr{H}_{\text {vac }}-\sqrt{g}\left(p g^{\#}+n h^{3} J_{\|} \otimes J_{\|}\right)$. Hence $\left(D_{g} \mathscr{H}\right)^{*} \cdot N$ $=\left(D_{g} \mathscr{H}_{\text {vac }}\right)^{*} \cdot N-N \sqrt{g}\left(p g^{\#}+n h^{3} J_{\|} \otimes J_{\|}\right)$. Computing in this fashion we find that (3.20) gives

$$
X_{H}(g, \pi)=\left(\begin{array}{l}
\left(D_{\pi} \mathscr{H}_{\mathrm{vac}}\right)^{*} \cdot N+\left(D_{\pi} \mathscr{J}_{\mathrm{vac}}\right)^{* \cdot} M \\
-\left(D_{g} \mathscr{H}_{\mathrm{vac}}\right)^{*} \cdot N-\left(D_{g} \mathscr{J}_{\mathrm{vac}}\right)^{*} \cdot M+N \sqrt{g}\left(p g^{\#}+n h^{3} J_{\|} \otimes J_{\|}\right)
\end{array}\right) .
$$

Therefore the evolution of $(g, \pi)$ is along the integral curves of $X_{H}$. Put another way, the evolution eqs. (3.4), (3.5) for $(g, \pi)$ are equivalent to the Poisson evolution equations $\dot{F}=\{F, H\}$ on $T^{*} \mathscr{M}$.

Our efforts are summarized in

Theorem 1. Let $H$ be the Hamiltonian given by (3.11). The following three conditions on a curve $(g, \pi, \mu, \eta, s)(t)$ are equivalent:

(i) It satisfies the evolution Eqs. (3.4) $\rightarrow(3.8)$.

(ii) It is an integral curve of the Hamiltonian vector field $X_{H}$ on $T^{*} \mathscr{M} \times s^{*}$ given by (1C.17) and (3.20).

(iii) It satisfies $\dot{F}=\{F, H\}$ where the Poisson bracket on $T^{*} \mathscr{M} \times s^{*}$ is the sum of the canonical bracket on $T^{*} \mathscr{M}$ (see (3.19)) and the +Lie-Poisson bracket on $s^{*}($ see (1C.15)).

The equations $\dot{F}=\{F, H\}$ may be regarded as the adjoint equations in the spirit of Fischer and Marsden (1979).

We now digress and explain why the seemingly artificial factor of 2 is necessary in the defining relations (3.1), (3.2) of $\mu$ and $\eta$. If this 2 were absent, then the key changes are: $(\tilde{\mu}, \tilde{\eta})=\left(n h J^{\perp} \sqrt{g}, n h^{3} J_{\|}^{\mathrm{b}} J^{\perp} \sqrt{g}\right), \quad \mathscr{H}=\mathscr{H}_{\mathrm{vac}}+2\left(J_{\|}^{i} \tilde{\mu}_{i}+\tilde{\eta}\right)\left(J^{\perp}\right)^{-1}-$ $2 p \sqrt{g}, \mathscr{J}=\mathscr{J}_{\text {vac }}-2 \tilde{\mu}$,

$$
\begin{aligned}
& \partial_{t} \tilde{\mu}=-\mathscr{L}_{\left(-M+N J_{\|}(J \perp)^{-1}\right)} \tilde{\mu}-\tilde{\eta} d\left(N\left(J^{\perp}\right)^{-1}\right)+(n N T \sqrt{g}) d s, \\
& \partial_{t} \tilde{\eta}=-\mathscr{L}_{\left(-M+N J_{\|}(J \perp)^{-1}\right)} \tilde{\eta},
\end{aligned}
$$

as well as

$$
\left(\frac{\partial H}{\delta \tilde{\mu}}, \frac{\delta H}{\delta \tilde{\eta}}, \frac{\delta H}{\delta s}\right)=2\left(-M+N J_{\|}\left(J^{\perp}\right)^{-1}, N\left(J^{\perp}\right)^{-1}, n N T \sqrt{g}\right) .
$$

Thus $H=\int_{\Sigma}(N \mathscr{H}+M \mathscr{J})$ no longer generates the evolution equations for $(\tilde{\mu}, \tilde{\eta}, s)$, though it still works for those of $(g, \pi)$. One could remedy the situation by replacing $T_{\alpha \beta}$ by $\frac{1}{2} T_{\alpha \beta}$ in the Einstein field equation, for then

$$
\begin{gathered}
\mathscr{H}=\mathscr{H}_{\mathrm{vac}}+\left(J_{\|}^{i} \tilde{\mu}_{i}+\tilde{\eta}\right)\left(J^{\perp}\right)^{-1}-p \sqrt{g}, \mathscr{J}=\mathscr{J}_{\mathrm{vac}}-\tilde{\mu}, \text { and hence } \\
\left(\frac{\partial H}{\delta \tilde{\mu}}, \frac{\delta H}{\delta \tilde{\eta}}, \frac{\partial H}{\delta s}\right)=\left(-M+N J_{\|}\left(J^{\perp}\right)^{-1}, N\left(J^{\perp}\right)^{-1}, n N T \sqrt{g}\right) ;
\end{gathered}
$$


on the other hand, the $\partial_{t}(\tilde{\mu}, \tilde{\eta})$ equations remain the same as $\left(^{*}\right)$ because they are obtained by a $(3+1)$ decomposition of ${ }^{(4)} \nabla \cdot T=0$, which is equivalent to ${ }^{(4)} \nabla \cdot\left(\frac{1}{2} T\right)=0$. Thus $H=\int_{\Sigma}(N \mathscr{H}+M \mathscr{J})$ will again generate the evolution equations for $(\tilde{\mu}, \tilde{\eta}, s)$; it will also generate those for $(g, \pi)$, since the source term in the $\partial_{t} \pi$ equation would have been halved by the $T_{\alpha \beta} \rightarrow \frac{1}{2} T_{\alpha \beta}$ process as well. This shows that, given our possibly prejudicial insistence that the Hamiltonian is always defined as $\int_{\Sigma}(N \mathscr{H}+M \mathscr{J})$ with $\mathscr{H}, \mathscr{J}$ given canonically by the prescription in (2.16), (2.17) (i.e. $-2 \sqrt{g}$ times the $\perp \perp$ and $\perp \|$ projection of Ein- $T$, respectively), the origin of the factor 2 is in the coupling between the fluid and the gravitational field.

Let us now turn to the issue of constraints and their preservation. If one specifies initial data on $\Sigma$, prescribes $(N, M)$ for all time, and then uses $(3.4) \rightarrow(3.8)$ to evolve the initial data into a curve $(g, \pi, \mu, \eta, s)(t)$, one obtains a fluid filled spacetime whose $(3+1)$ description is $(g, \pi, \mu, \eta, s, N, M)(t)$. However, in order that the fluid and the spacetime obtained satisfy all components of the Einstein field equation, the initial data must be constrained by (3.9) and (3.10). As a matter of consistency, we need to check that the constraints are preserved by the evolution Eqs. (3.4) $\rightarrow$ (3.8). A (long) calculation shows that such is indeed the case, for

$$
\begin{aligned}
\partial_{t} \mathscr{J} & =\mathscr{L}_{M} \mathscr{J}+\mathscr{H} d N, \\
\partial_{t} \mathscr{H} & =\mathscr{L}_{M} \mathscr{H}+N^{-1} \nabla \cdot\left(N^{2} \mathscr{J}\right) .
\end{aligned}
$$

Some key ingredients that help establish (3.24), (3.25), are

$$
\begin{gathered}
\left(D_{g} \mathscr{H}_{\mathrm{vac}}\right) \cdot\left(D_{\pi} \mathscr{H}_{\mathrm{vac}}\right)^{*} \cdot N-\left(D_{\pi} \mathscr{H}_{\mathrm{vac}}\right) \cdot\left(D_{g} \mathscr{H}_{\mathrm{vac}}\right)^{*} \cdot N=N^{-1} \nabla \cdot\left(N^{2} \mathscr{J}_{\mathrm{vac}}\right) \\
\left(D_{g} \mathscr{J}_{\mathrm{vac}}\right) \cdot\left(D_{\pi} \mathscr{H}_{\mathrm{vac}}\right)^{*} \cdot N-\left(D_{\pi} \mathscr{J}_{\mathrm{vac}}\right) \cdot\left(D_{g} \mathscr{H}_{\mathrm{vac}}\right)^{*} \cdot N=\mathscr{H}_{\mathrm{vac}} d N \\
\left(D \mathscr{J}_{\mathrm{vac}}\right)^{*} \cdot X=\left(-\mathscr{L}_{X} \pi, \mathscr{L}_{X} g\right) \\
\left(D_{\pi} \mathscr{J}_{\mathrm{vac}}\right) \cdot \omega=-2 \nabla \cdot \omega
\end{gathered}
$$

Their verification can be found in Fischer and Marsden (1979) (caution: our $\mathscr{J}_{\text {vac }}=$ - their $\mathscr{J}_{\text {vac }}$, and our $M=-$ their $M$ ).

We close this section with a geometrical description of the formalism we have used. This will be useful in Sect. 4.

Denote $\left(g, \pi, J^{\perp}, J_{\|}, s\right)$-space by $T^{*} \mathscr{M} \times V$ and, as above, $(g, \pi, \mu, \eta, s)$-space by $T^{*} \mathscr{M} \times s^{*}$. Define the map

by

$$
\Omega: T^{*} \mathscr{M} \times V \rightarrow T^{*} \mathscr{M} \times s^{*}
$$

$$
\Omega\left(g, \pi, J^{\perp}, J_{\|}, s\right)=\left(g, \pi, 2 n h^{3} J_{\|}^{\mathrm{b}} J^{\perp} \sqrt{g}, 2 n h J^{\perp} \sqrt{g}, s\right) .
$$

Let $D$ be the submanifold in $T^{*} \mathscr{M} \times V$ on which the non-zero inertial mass condition (1A.4) and the causality condition (1A.11) are valid. The reasoning leading to (3.3) shows that $\Omega_{\mid D}: D \rightarrow \Omega(D)$ is a local diffeomorphism. Let $C$ be the submanifold in $T^{*} \mathscr{M} \times V$ defined by the constraints (2.14), (2.15). The system of evolution Eqs. (2.12), (2.13), (2.26), (2.20), and (2.27) maps $C$ into $C$ for all times. On the other hand, the constraints (3.9), (3.10) describe the submanifold $\Omega(C \cap D)$. Thus, we can phrase things as: the system of evolution Eqs. (3.4) $\rightarrow(3.8)$ is defined on $\Omega(D)$ and the evolution operator maps $\Omega(C \cap D)$ into $\Omega(C \cap D)$. 


\section{Another Viewpoint}

In this section we describe, following Walton (1980), an approach in which the gauge Lie algebra $\mathscr{X} \times \mathscr{F}$ is parametrized by $\bar{M}$ and $\bar{N}$ (see Sect. 1B) rather than the usual shift vector field and the lapse function.

Define $\mu$ and $\eta$ as before:

$$
\begin{aligned}
& \mu=2 n h^{3} J_{\|}^{b} J^{\perp} \sqrt{g}, \\
& \eta=2 n h J^{\perp} \sqrt{g} .
\end{aligned}
$$

Our basic variables are $(g, \pi, \mu, \eta, s)$; therefore the phase space is again $\left(T^{*} \mathscr{M} \times s^{*},\{\}\right.$ +\{\}$\left._{+}\right)$. The evolution equations are

$$
\begin{aligned}
\partial_{t} g= & \left(D_{\pi} \mathscr{H}_{\mathrm{vac}}\right)^{*} \cdot\left(\bar{N} J^{\perp}\right)+\left(D_{\pi} \mathscr{J}_{\mathrm{vac}}\right)^{*}\left(\bar{N} J_{\|}-\bar{M}\right), \\
\partial_{t} \pi= & -\left(D_{g} \mathscr{H}_{\mathrm{vac}}\right)^{*}\left(\bar{N} J^{\perp}\right)-\left(D_{g} \mathscr{J}_{\mathrm{vac}}\right)^{*}\left(\bar{N} J_{\|}-\bar{M}\right) \\
& +\sqrt{g}\left(\bar{N} J^{\perp}\right)\left(n h^{3} J_{\|} \otimes J_{\|}+p g^{\#}\right), \\
\partial_{t} \mu= & -\mathscr{L}_{\bar{M}} \mu-\eta d \bar{N}+\left(2 n \bar{N} J^{\perp} T \sqrt{g}\right) d s, \\
\partial_{t} \eta= & -\mathscr{L}_{\bar{M}} \eta, \\
\partial_{t} s= & -\mathscr{L}_{\bar{M}} s,
\end{aligned}
$$

where, in contradistinction to Sect. $3,\left(J^{\perp}, J_{\|}\right)$are regarded here as functionals of $(g, \pi, s)$ obtained by solving, for each fixed $s$, certain projections of the Einstein field equation, namely

$$
\begin{array}{r}
\mathscr{H}\left(g, \pi, J^{\perp}, J_{\|}, s\right)=\mathscr{H}_{\text {vac }}+2 n h^{3}\left(J^{\perp}\right)^{2} \sqrt{g}-2 p \sqrt{g}=0, \\
\mathscr{J}\left(g, \pi, J^{\perp}, J_{\|}, s\right)=\mathscr{J}_{\text {vac }}-2 n h^{3} J^{\perp} J_{\|}^{\mathrm{b}} \sqrt{g}=0 .
\end{array}
$$

Equations (4.8) and (4.9) were previously known as the constraints, but here they are to be used as identities (since they are solved) and the name constraint could be misleading. Following Moncrief (1977), we see that (4.8) and (4.9) can indeed be solved for

$$
\left(J^{\perp}, J_{\|}\right)=\left(J^{\perp}, J_{\|}\right)(g, \pi s),
$$

provided that the non-zero inertial mass assumption and a causality condition (respectively (1A.4) and (1A.11)) are both satisfied.

Let us explain geometrically what has been done so far, using the language introduced at the end of the last section.

Using (4.8) and (4.9) as identities is the statement that in $T^{*} \mathscr{M} \times V$, we have restricted the system of evolution Eqs. (2.12), (2.13), (2.26), (2.20) and (2.27) to the constraint submanifold $C$. Having (4.10) means that such a system is further restricted to $C \cap D$, and local coordinates $(g, \pi, s)$ are being used, consequently reducing the system to an explicitly unconstrained one. Transplanting this unconstrained system from $C \cap D$ to $\Omega(C \cap D)$, we obtain some system of evolution equations, say "*”. The collection of evolution Eqs. (4.3) $\rightarrow(4.7)$ represents one of possibly many systems on $T^{*} \mathscr{M} \times \diamond^{*}$ which, when consistently restricted onto $\Omega(C \cap D)$, agrees with the system “*”. Now, since points $(g, \pi, \mu, \eta, s)$ in $\Omega(C \cap D)$ are 
characterized by the conditions

$$
\alpha:=\mu-\left(2 n h^{3} J_{\|}^{b} J^{\perp} \sqrt{g}\right)(g, \pi, s)=0,
$$

and

$$
\beta:=\eta-\left(2 n h J^{\perp} \sqrt{g}\right)(g, \pi, s)=0,
$$

and since (4.3) $\rightarrow(4.7)$ is supposed to restrict consistently to $\Omega(C \cap D)$, we must check that the quantities $\alpha$ and $\beta$ are preserved by the evolution Eqs. (4.3) $\rightarrow$ (4.7). A (long) calculation shows that

$$
\begin{aligned}
& \partial_{t} \alpha=-\mathscr{L}_{\bar{M}} \alpha-\beta d \bar{N}, \\
& \partial_{t} \beta=-\mathscr{L}_{\bar{M}} \beta .
\end{aligned}
$$

So, if the conditions (4.11) and (4.12) are satisfied initially, they are satisfied for all time.

We next study the Hamiltonian structure of the system (4.3) $\rightarrow(4.7)$. We shall show that it is generated by the Hamiltonian

$$
\bar{H}=\bar{H}(g, \pi, \mu, \eta, s, \bar{N}, \bar{M})=\int_{\Sigma}(\bar{N} \bar{H}+\bar{M} \overline{\mathscr{J}})
$$

where

$$
\begin{aligned}
\overline{\mathscr{H}}(g, \pi, \eta, s) & =J^{\perp} \mathscr{H}_{\mathrm{vac}}+J_{\|}^{i}\left(\mathscr{J}_{\mathrm{vac}}\right)_{i}-2 J^{\perp} p \sqrt{g}+\eta, \\
\overline{\mathscr{J}}(g, \pi, \mu) & =-\mathscr{J}_{\mathrm{vac}}+\mu .
\end{aligned}
$$

The use of (4.10) is understood.

Let us indicate how the expression for $\bar{H}$ is obtained. Note first that even though the quantities $\mathscr{H}\left(g, \pi, J^{\perp}, J_{\|}, s\right)=\mathscr{H}_{\text {vac }}+2 n h^{3}\left(J^{\perp}\right)^{2} \sqrt{g}-2 p \sqrt{g}$ and $\mathscr{J}\left(g, \pi, J^{\perp}, J_{\|}, s\right)$ $=\mathscr{J}_{\text {vac }}-2 n h^{3} J^{\perp} J_{\|}^{b} \sqrt{g}$ are now identically zero by virtue of (4.10), their rewrites using the defining relations (4.1), (4.2) at "appropriate" places, namely

$$
\begin{aligned}
\tilde{H}(g, \pi, \mu, \eta, s) & =\mathscr{H}_{\mathrm{vac}}+\left(J_{\|}^{i} \mu_{i}+\eta\right)\left(J^{\perp}\right)^{-1}-2 p\left(h\left(J^{\perp}, J_{\|}, g\right), s\right) \sqrt{g}, \\
\tilde{J}(g, \pi, \mu) & =\mathscr{J}_{\mathrm{vac}}-\mu,
\end{aligned}
$$

are generally non-vanishing functionals defined on the phase space $T^{*} \mathscr{M} \times s^{*}$. In (4.18) and (4.19), the $\left(J^{\perp}, J_{\|}\right)$are functionals of $(g, \pi, s)$ by (4.10). Now, using (1B.14) (1B.15),

$$
\int_{\Sigma}(N \tilde{\mathscr{H}}+M \tilde{\mathscr{J}})=\int_{\Sigma}\left(\bar{N} J^{\perp}\right) \tilde{\mathscr{H}}+\left(\bar{N} J_{\|}-\bar{M}\right) \tilde{\mathscr{J}}=\int_{\Sigma} \bar{N}\left(J^{\perp} \tilde{\mathscr{H}}+J_{\|} \tilde{\mathscr{J}}\right)+\bar{M}(-\tilde{\mathscr{J}}) .
$$

It is obvious that $J^{\perp} \tilde{\mathscr{H}}+J_{\|} \tilde{\mathscr{J}}$ and $-\tilde{\mathscr{J}}$ are simply the $\overline{\mathscr{H}}$ and $\overline{\mathscr{J}}$ defined in (4.16) and (4.17)

Let us make one more observation which will be needed. Note that the functional

$$
\overline{\mathscr{H}}^{\prime}\left(g, \pi, \eta, s, J^{\perp}, J_{\|}\right)=J^{\perp} \mathscr{H}_{\text {vac }}+J_{\|} \mathscr{J}_{\text {vac }}-2 J^{\perp} p\left(h\left(J^{\perp}, J_{\|}, g\right), s\right) \sqrt{g}+\eta
$$

is, upon the use of (4.10), numerically equal to the $\overline{\mathscr{H}}(g, \pi, \eta, s)$ defined in (4.16). Using 
(4.8) and (4.9) (equivalently (4.10)), is it easy to verify that

$$
\begin{gathered}
\partial_{J^{\perp}} \overline{\mathscr{H}}^{\prime}=\mathscr{H}\left(g, \pi, J^{\perp}, J_{\|}, s\right)=0, \\
\partial_{J_{\|}} \overline{\mathscr{H}}^{\prime}=\mathscr{J}\left(g, \pi, J^{\perp}, J_{\|}, s\right)=0 .
\end{gathered}
$$

We are now ready to show that $\bar{H}$, regarded as a function on $\left(T^{*} \mathscr{M},\{\}\right)$, generates the evolution equations of $(g, \pi)$. As before, the Hamiltonian vector field $X_{\bar{H}}$ of $\bar{H}$ is

$$
x_{\bar{H}}(g, \pi)=\left(\begin{array}{cc}
0 & 1_{6 \times 6} \\
-1_{6 \times 6} & 0
\end{array}\right)\left(\begin{array}{l}
\left(D_{g} \overline{\mathscr{H}}\right)^{*} \cdot \bar{N}+\left(D_{g} \overline{\mathscr{J}}\right)^{*} \cdot \bar{M} \\
\left(D_{\pi} \overline{\mathscr{H}}\right)^{*} \cdot \bar{N}+\left(D_{\pi} \overline{\mathscr{J}}\right)^{*} \cdot \bar{M}
\end{array}\right) .
$$

By (4.21) and (4.22), we have

$$
\begin{aligned}
D_{g} \overline{\mathscr{H}}=J^{\perp} D_{g} \mathscr{H}_{\mathrm{vac}} & +J_{\|} D_{g} \mathscr{J}_{\mathrm{vac}}-2 J^{\perp} \partial_{g}\left[\sqrt{g} p\left(h\left(J^{\perp}, J_{\|}, g\right), s\right)\right] \\
& +\left(\partial_{J \perp} \overline{\mathscr{H}}^{\prime}\right) D_{g} J^{\perp}+\left(\partial_{J_{\|}} \overline{\mathscr{H}}^{\prime}\right) D_{g} J_{\|} \\
= & J^{\perp} D_{g} \mathscr{H}_{\mathrm{vac}}+J_{\|} D_{g} \mathscr{J}_{\mathrm{vac}}-J^{\perp} \sqrt{g}\left(n h^{3} J_{\|} \otimes J_{\|}+g^{\#} p\right) .
\end{aligned}
$$

Hence

$$
\left(\bar{D}_{g} \overline{\mathscr{H}}\right)^{*} \cdot \bar{N}=\left(D_{g} \mathscr{H}_{\mathrm{vac}}\right)^{*} \cdot\left(\bar{N} J^{\perp}\right)+\left(D_{g} \mathscr{J}_{\mathrm{vac}}\right)^{*} \cdot\left(\bar{N} J_{\|}\right)-\left(\bar{N} J^{\perp}\right) \sqrt{g}\left(n h^{3} J_{\|} \otimes J_{\|}+g^{\#} p\right) .
$$

Computing this way, one can check that (4.23) is explicitly

$$
x_{H}(\dot{g}, \pi)=\left(\begin{array}{c}
\text { right-hand-side of }(4.3) \\
\text { right-hand-side of }(4.4)
\end{array}\right) .
$$

Therefore the evolution of $(g, \pi)$ is along the integral curves of $X_{\bar{H}}$.

Next we regard $\bar{H}$ as a function on $\left(s^{*},\{\}_{+}\right)$. The use of (4.21) and (4.22) as above will also tell us that

$$
\left(\frac{\delta \bar{H}}{\delta \mu}, \frac{\delta \bar{H}}{\delta \eta}, \frac{\delta \bar{H}}{\delta s}\right)=\left(\bar{M}, \bar{N}, 2 n \bar{N} J^{\perp} T \sqrt{g}\right),
$$

so by Proposition 1 (Sect. 1C), we see that the evolution of $(\mu, \eta, s)$ is along the integral curves of $X_{\mathscr{H}}$.

Theorem 2. Let $\bar{H}$ be the Hamiltonian given in (4.15). The following conditions on a curve $(g, \pi, \mu, \eta, s)(t)$ are equivalent

(i) it satisfies the evolution Eqs. (4.3) $\rightarrow(4.7)$;

(ii) it is an integral curve of the Hamiltonian vector field $X_{\bar{H}}$ on $\left(T^{*} \mathscr{M} \times s^{*}\right.$, \{\}$+\{\}+)$

(iii) it satisfies $\dot{F}=\{F, \bar{H}\}$ where the Poisson bracket is the sum of the canonical bracket and the + Lie-Poisson bracket.

Finally, we turn to the issue of constraints. Since our Hamiltonian is $\bar{H}=$ $\int_{\Sigma}(\bar{N} \overline{\mathscr{H}}+\bar{M} \overline{\mathscr{J}})$ and $\bar{N}, \bar{M}$ are atlas fields, we expect the following constraints on the initial data $(g, \pi, \mu, \eta, s)$ :

$$
\overline{\mathscr{H}}(g, \pi \eta, s)=J^{\perp} \mathscr{H}_{\mathrm{vac}}+J_{\|} \mathscr{J}_{\mathrm{vac}}-2 J^{\perp} p \sqrt{g}+\eta=0,
$$




$$
\overline{\mathscr{J}}(g, \pi, \mu)=-\mathscr{J}_{\mathrm{vac}}+\mu=0 .
$$

Using (4.8) and (4.9), one can show that (4.26) and (4.27) can be re-expressed, respectively, as none other than the conditions (4.11) and (4.12) which characterize the submanifold $\Omega(C \cap D)$. As was shown in (4.13) and (4.14), such conditions are preserved by the evolution Eqs. (4.3) $\rightarrow(4.7)$.

\section{Comments}

In this paper we have shown that the Einstein-Euler equations for a perfect fluid coupled to gravity are Hamiltonian. The key features of our Hamiltonian structure are: (a) it is valid for any lapse and shift, i.e. for any gauge condition; (b) it utilizes LiePoisson brackets for the fluid variables, which automatically encode the Lin constraints; (c) the Hamiltonian is explicitly linear in the lapse and shift, while the Poisson brackets are independent of them; and (d) the constraints are not solved, and the system is explicit. An important step that allows this to succeed is the correct choice $(\mu, n)$ of momentum and density variables for the fluid.

In what follows, we elaborate on a few points to make further contact with the literature and to point out a few directions for future research. In doing so, the exposition will draw heavily on the existing literature and, unlike the main text, makes no attempt to be self-contained. For expositional simplicity we shall drop the dependence on the entropy $s$, since it is just advected and plays no role in the points we wish to raise.

A. Lagrangian (Material) Descriptions. The original variational principle of Taub (1948) in which world lines are varied is in the spirit of a Lagrangian description. In our approach, the dynamic phase space (dropping the entropy) is

$$
T^{*} \mathscr{M} \times(\mathscr{X} \times \mathscr{F})^{*},
$$

where $\mathscr{M}$ is the space of Riemannian metrics on a model 3-hypersurface $\Sigma, \mathscr{X}$ is the space of vector fields on $\Sigma$ and $\mathscr{F}$ is the space of functions. The Poisson bracket is the Lie-Poisson bracket on the dual of the semidirect product $\mathscr{X} \propto \mathscr{F}$. As explained in Marsden et al. (1984a, b), there are a number of ways of obtaining $(\mathscr{X} \times \mathscr{F})^{*}$ from a Lagrangian description by a Lagrangian to Eulerian map (i.e. by reduction).

One can start with

$$
T^{*} \mathscr{M} \times T^{*}(\operatorname{Diff} \times \mathscr{F})
$$

as a Lagrangian phase space and pass to (5A.1) by passing from the group Diff $\times \mathscr{F}$ to its Lie algebra $\mathscr{X} \times \mathscr{F}$. (This does not presuppose any semidirect structure; as it is shown, this follows.) The canonical bracket on (5A.2) has a Lagrangian description: $\phi \in$ Diff represents a spatial fluid configuration (ultimately placed in spacetime by means of the slicing) and $\eta_{\mathrm{Ref}} \in \mathscr{F}^{*}$ represents a reference density - transformed to $\eta$ when we pass to (5A.1). This Lagrangian approach appears to be similar to Kunzle and Nester (1984).

Notice that (5A.2) contains $\mathscr{F} \times \mathscr{F} *$, so a (cyclic) variable conjugate to the density is present. This variable is related to time translation along fluid trajectories. This is analogous to the thermasy function of van Dantzig (1939), a variable 
conjugate to entropy. Similarly, in MHD, the variable conjugate to the magnetic field is the polarization; cf. Calkin (1963). However, the extra factor is a cyclic variable so one directly passes from (5A.2) to

$$
T^{*} \mathscr{M} \times\left(T^{*} \operatorname{Diff} \times \mathscr{F} *\right)
$$

by reduction by $\mathscr{F}$ (see Marsden et al., (1984b) for the general theory for this). This is related to the (optional) use of cyclic variables by, for example, Schutz (1970) and Walton $(1979,1980)$

One can also start with a Lagrangian phase space

$$
T^{*}[(\text { Metrics } \times \text { Functions) over Diffeomorphisms }],
$$

where one thinks of the metrics as being dragged by the fluid diffeomorphisms. This possibility was foreshadowed by Fischer and Marsden (1972) but with a different point of view. The approach (5A.4) more closely resembles the lapse and shift $\bar{N}, \bar{M}$ tied to particle paths that were used in Sect. 4 and in Walton (1980).

The approach of Moncrief (1977) and Demaret and Moncrief (1980) is a further reduction of (5A.1). The constraints $\mathscr{H}=0, \mathscr{J}=0$ can be viewed as the zero level set of the momentum map for the action of ${ }^{(4)}$ Diff, the four dimensional diffeomorphism group, thought of as a gauge group. Then by general reduction theory (Marsden and Weinstein, (1974)) the set $(\mathscr{H}=0, \mathscr{J}=0) /{ }^{(4)}$ Diff carries the dynamics; one more reduction by $\operatorname{Diff} \propto \mathscr{F}$ (the particle relabling group) gives black $T^{*} \mathscr{M}$ as the reduced space. This approach has the disadvantage of having implicit dependence (the fluid variables are eliminated) but the advantage of fewer degrees of freedom.

It would be quite interesting to develop a covariant multisymplectic (or Poisson) formalism that leads in a natural way from the covariant formalism to the $3+1$ formalism (Lagrangian, Eulerian or mixed).

B. Clebsch Potentials. The approach of Schutz $(1970,1971)$ is to develop the Hamiltonian formalism using the classical Clebsch potentials in the spirit of Seliger and Whitham (1968). This formalism is consistent with and in fact follows from what we have developed. (The formula (33) of Schutz (1971) was a crucial tip for our approach.) Indeed, the brackets for (5A.1) are independent of lapse and shift and are, in the fluid variables, identical to the brackets for the non-relativistic case. Since Clebsch variables are known to apply no matter what the physical interpretation is (see Marsden and Weinstein, (1983); Marsden et al., (1984a) for the general theory of Clebsch potentials), they can be applied to the brackets for (5A.1). The Schutz formalism results.

C. Lin Constraints. Any solution of Lie-Poisson equations leaves the coadjoint orbits invariant. These orbits represent sets of momentum and density configurations that are kinematically reachable one from the other. They are symplectic manifolds, so standard variational principles are valid when varied paths are constrained to lie in them. Thus, from Seliger and Whitham (1968), Bretherton (1970), Marsden and Weinstein (1983), or Marsden et al. (1984a, b), we see that the Lin constraint sets are identical to the coadjoint orbits. In the Lie-Poisson formalism these constraints need not be imposed; the brackets automatically ensure their preservation by the equations. Of course the use of Clebsch potentials is 
another mechanism for building in the constraints; it has the obvious disadvantage that it deals with non-physical fields and some fields (having knotted vortex lines for example) cannot be written in terms of Clebsch potentials.

D. Electromagnetic Fields. Given the non-relativistic results (especially Morrison and Greene, (1980); Marsden and Weinstein, (1982); Spencer, (1982); Marsden and Weinstein, (1983); Holm and Kupershmidt, (1983), Marsden et al., (1984a)), and the results of this paper, it now seems that one can readily proceed to obtain brackets and Hamitonian formalisms for a wide variety of field theories coupled to gravity, such as charged relativistic fluids, relativistic MHD, and relativistic plasmas. For relativistic plasmas, we recall from Marsden and Weinstein (1982) that if the electromagnetic variables $(E, A)$ are used, the bracket should be just that of

$$
T^{*} \mathscr{M} \times T^{*} \mathfrak{a} \times s^{*}
$$

where $\mathfrak{a}$ is the space of $A$ 's and $s^{*}$ is the space of (plasma) densities on the mass shell of $T^{*}$ (Spacetime). When the system is reduced to the fields $(E, B)$ by the electromagnetic gauge group, there is an important but complicated coupling in the bracket between the electromagnetic fields and the plasma field (see Spencer, (1982), Marsden et al. (1983)). It would be of interest to see how the gravitational field complicates this story.

E. Linearization Stability. The basic results on linearization stability link solutions with symmetry to singularities in the space of solutions (Fischer et al., (1980); Arms et al., 1981, 1982). The methods depend crucially on the dynamic Hamiltonian formalism. Once the constraints are identified and the evolution equations are written in the adjoint form of Fischer and Marsden (1979), the linearization stability program can be carried out (see, for example, Arms, 1977, 79, 81; Bao, 1983, 1984; Bao et al., 1984; Joshi and Saraykar, 1981).

Fluids, however, have a complication. Namely, the brackets are degenerate. However, if we restrict our allowed fields to the coadjoint orbits (see C. above) the bracket becomes nondegenerate, so the connection between symmetries and linearization instabilities should be reinstated. Such is indeed found to be the case and will be reported in a future publication.

This does not contradict the remarks of D'Eath (1974) or of Arms (1979*). For example, D'Eath argues that since the constraints can be eliminated, there can never be any linearization instabilities. However, to do so requires variations of the fields that violate the Lin constraints.

We expect that our approach will be of use in the perturbation theory of matter filled spacetimes by dividing the perturbations into two pieces: the ones consistent with the Lin constraints (where there will be linearization instabilities) and the ones orthogonal to them (that change the total baryon number, for example).

F. Dynamic Stability. The Lie-Poisson formalism has been remarkably successful in the nonrelativistic case in establishing nonlinear dynamic stability results for fluid systems. See, for example, Arnold (1966b); Holm et al. (1983, 84); Abarbanel et al. (1984); Hazeltine et al. (1984). These results depend primarily on special conserved quantities for the fluid variables that are associated to the coadjoint orbits. Since the relativistic brackets are the same, these Casimirs are also conserved for general 
relativistic fluids - even for a general lapse and shift. It is therefore reasonable to apply these methods to the general relativistic case. A good example to begin with might be spherically symmetric neutron star cores (see Moncrief, (1974)). Of course the dynamic stability of the gravitational fields will probably be more difficult to deal with.

Acknowledgements. Some initial discussions on this problem with Vince Moncrief were crucial to the success of our endeavors. We are greatly indebted to him. We also thank Judy Arms, Darryl Holm, Bob Jantzen, Richard Montgomery, Phil Morrison, Tudor Ratiu and Abe Taub for their interest and helpful comments.

\section{References}

Abarbanel, H., Holm, D., Marsden, J., Ratiu, T.: [1984]: Nonlinear stability of stratified flow (to appear) Arms, J.: Linearization stability of the Einstein-Maxwell system, J. Math. Phys. 18, 830-833 (1977)

Arms, J.: Linearization stability of gravitational and gauge fields. J. Math. Phys. 20, 443-453 (1979)

Arms, J.: [1979*]: Does matter break the link between symmetry and linearization instability?, GRG essay (unpublished)

Arms, J.: The structure of the solution set for the Yang-Mills equations. Math. Proc. Camb. Phil Soc. 90, $361-372(1981)$

Arms, J., Marsden, J., Moncrief, V.: Bifurcations of momentum mappings. Commun. Math. Phys. 78, 455-478 (1981)

Arms, J., Marsden, J., Moncrief, V.: The structure of the space of solutions of Einstein's equations, II: Several Killing fields and the Einstein Yang-Mills equations, Ann. Phys. 144, 81-106 (1982)

Arnold, V.: Sur la géometrie differentielle des groupes de Lie de dimension infinie et ses applications a l'hydrodynamique des fluids parfaits. Ann. Inst. Fourier, Grenoble, 16, 319-361 (1966a)

Arnold, V.: [196jb]: An a priori estimate in the theory of hydrodynamic stability. Am. Math. Soc. Transl. 19, 267-269 (1969)

Arnowitt, R., Deser, S., Misner, C. W.: The dynamics of general relativity. In Gravitation: an introduction to current research. pp. 227-65. Witten, L. (ed.) New York: Wiley 1962

Bao, D.: Some aspects in the dynamics of supergravity. Thesis, U.C. Berkeley 1983

Bao, D.: A sufficient condition for the linearization stability of $N=1$ supergravity: A preliminary report. Ann. Phys 158, 211-278 (1984)

Bao, D., Isenberg, J., Yasskin, P.: [1984]: The dynamics of the Einstein-Dirac system I: A principal bundle formulation of the theory and its canonical analysis. Ann. Phys. (to appear)

Bialynicki-Birula, I., Hubbard, J.C., Turski, L. A.: Gauge-independent canonical formulation of relativistic plasma theory (preprint)

Bretherton, F. P.: A note on Hamilton's principle for perfect fluids. J. Fluid Mech. 44, 19-31 (1970)

Calkin, M. G.: An action principle for magnetohydrodynamics, Can. J. Phys. 41, 2241-2251 (1963)

Carter, B.: Elastic perturbation theory in general relativity and a variational principle for a rotating solid star. Commun. Math. Phys. 30, 261-286 (1973)

D'Eath, P.: Three perturbation problems in general relativity, Thesis, Cambridge. Ann. Phys. 98, 237-263 (1974)

Demaret, J., Moncrief, V.: Hamiltonian formalism for perfect fluids in general relativity. Phys. Rev. D21, $2785-2793(1980)$

Dirac, P. A. M.: Fixation of coordinates in the Hamiltonian theory of gravitation. Phys. Rev. 114, 924-30 (1959)

Dirac, P. A. M.: Lectures on quantum mechanics. Belfer graduate school of science, Monograph Series No. 2. New York: Yeshiva University 1964

Dzyaloshinskii, I. E., Volovick, G. E.: Poisson brackets in condensed matter physics. Ann. Phys. 125, 6797 (1980)

Fischer, A., Marsden, J.: General relativity as a dynamical system on the manifold $\mathscr{A}$ of riemannian metrics which cover diffeomorphism, In: Methods of local and global differential geometry in general relativity. Lecture Notes in Physics, pp. 176-188 Vol. 14, Berlin, Springer, Heidelberg, New York: 1972 
Fischer, A., Marsden, J.: Topics in the dynamics of general relativity. In: Isolated gravitating systems in general relativity, Ehlers, J. (ed.), Ital. Phys. Soc. 322-395 (1979)

Fischer, A., Marsden, J., Moncrief, V.: The structure of the space of solutions of Einstein's equations. I. One Killing Field. Ann. Inst. H. Poincaré. 33, 147-194 (1980)

Guillemin, V., Sternberg, S.: The moment map and collective motion, Ann Phys. 127, 220-253 (1980)

Hanson, A., Regge, T., Teitelboim, C.: Constrained Hamiltonian systems. Accad. Nazionale Lincei, Rome. No. 22, 1-35 (1976)

Hawking, S. W., Ellis, G. F. R.: The large scale structure of spacetime. Cambridge: Cambridge University Press 1973

Hazeltine, R. D., Holm, D. D., Marsden, J. E., Morrison, P. J.: Generalized Poisson brackets and nonlinear Liapunov stability-application to reduced MHD Proc. Plasma Phys. Conf., Lausanne, June 1984

Holm, D. D., Kupershmidt, B. A.: Poisson brackets and Clebsch representations for magnetohydrodynamics, multifluid plasmas, and elasticity. Physica 6D, 347-363 (1983)

Holm, D. D., Kupershmidt, B. A.: Relativistic fluid dynamics as a hamiltonian system. Phys. Lett. 101A, 23-26 (1984)

Holm, D. D., Marsden, J. E., Ratiu, T., Weinstein, A.: Nonlinear stability conditions and a priori estimates for barotropic hydrodynamics. Phys. Lett. 98A, 15-21 (1983)

Holm, D., Marsden, J., Ratiu, T., Weinstein, A.: [1984]: Nonlinear stability of equilibria in fluid and plasma systems. Phys. Rep. (to appear)

Isenberg, J.: The construction from initial data of spacetimes with nontrivial spatial and bundle topology. Ann. Phys. 129, 223-248 (1980)

Joshi, N., Saraykar, R.: Linearization stability of Einstein equations coupled with self-gravitating scalar fields. J. Math. Phys. 22, 343-347 (1981)

Kunzle, H. P., Nester, J. M.: Hamiltonian formulation of gravitating perfect fluids and the Newtonian limit. J. Math. Phys. 25, 1009-1018 (1984)

Lichnerowicz, A.: Relativistic hydrodynamics and magnetohydramics: Lectures on the existence of solutions. New York: W. A. Benjamin 1967

Lund, F., Canonical quantization of relativistic balls of dust. Phys. Rev. D, 8, 3253 (1973)

Marsden, J., Ratiu, T., Weinstein, A.: [1984a]: Semidirect products and reduction in mechanics. Trans. Am. Math. Soc. 281, 147-177 (1984)

Marsden, J., Ratiu, T., Weinstein, A.: [1984b]: Reduction and Hamiltonian structures on duals of semidirect product Lie algebras. Cont. Math. AMS 28 (1984)

Marsden, J., Weinstein, A.: Reduction of symplectic manifolds with symmetry. Rep. Math. Phys. 5, 121130 (1974)

Marsden, J., Weinstein, A.: The Hamiltonian structure of the Maxwell-Vlasov equations. Physica 4D, 394-406 (1982)

Marsden, J. E., Weinstein, A.: Coadjoint orbits, vortices and Clebsch variables for incompressible fluids. Physica 7D, 305-323 (1983)

Marsden, J. E., Weinsten, A., Ratiu, T., Schmid, R., Spencer, R. G.: Hamiltonian systems with symmetry, coadjoint orbits and plasma physics, Proc,. IUTAM-ISIMM Symposium on: Modern developments in analytical mechanics. Torino, June 7-11, 1982. Atti Accad. Sci. Torino 117, 289-340 (1983)

Misner, C. W., Thorne, K., Wheeler, J. A.: Gravitation, San Francisco: W. H. Freeman 1973

Moncrief, V.: Gravitational perturbations of spherically symmetric systems, II. Perfect fluid interiors. Ann. Phys. 88, 343-370 (1974)

Moncrief, V.: Hamiltonian formalism for relativistic perfect fluids. Phys. Rev. D. 16, 1702-1705 (1977)

Morrison, P. J., Greene, J. M.: Noncanonical Hamiltonian density formulation of hydrodynamics and ideal magnetohydrodynamics. Phys. Rev. Lett. 45, 790-794 (1980)

Ratiu, T.: Euler-Poisson equations on Lie algebras. Thesis, U.C. Berkeley 1980

Schutz, B. F.: Perfect fluids in general relativity-velocity potentials and a variational principle. Phys. Rev. D2, 2762-2773 (1970)

Schutz, B. F.: The Hamiltonian theory of a relativistic perfect fluid. Phys. Rev. D4, 3559-3566 (1971)

Schutz, B. F., Sorkin, R.: Variational aspects of relativistic field theories, with application to perfect fluids. Ann. Phys. 107, 1-43 (1977)

Seliger, R. L., Whitham, G. B.: Variational principles in continuum mechanics. Proc. Roy. Soc. 305, 1-25 (1968) 
Smarr, L., Taubes, C., Wilson, J. [1980]: General relativistic hydrodynamics. In: The comoving, Eulerian and velocity potential formalisms. Essays on General Relativity, pp. 157-183, Tipler, F. (ed.) New York: Academic Press

Spencer, R. G.: The Hamiltonian structure of multi-species fluid electrodynamics. In: Mathematical methods in hydrodynamics and integrability in related dynamical systems. Tabor, M., Treve, Y. M., (eds.), AIP Conf. Proc., La Jolla Institute 1981, 88, 121-126 (1982)

Tam, K. K.: A variational principle in relativistic magnetohydrodynamics. Can. J. Phys. 44, 2403-2409 (1966)

Tam, K. K., O'Hanlon, J.: Relativistic magnetohydrodynamics of a gravitating fluid. Il Nuovo Cimento 62B, 351-359 (1969)

Taub, A. H.: Relativistic Rankine-Hugoniot equations. Phys. Rev. 74, 328-334 (1948)

Taub, A. H.: General relativistic variational principle for perfect fluids. Phys. Rev. 94, 1468-1470 (1954)

Taub, A. H.: Stability of general relativistic gaseous masses and variational principles. Commun. Math. Phys. 15, 235-254 (1969)

Taub, A. H.: Variational principles and relativistic magnetohydrodynamics. Colloq. CNRS, 184, 189$200(1970)$

Taub, A. H.: Variational principles in general relativity, Bressanone lectures, pp. 206-300. Rome: Centro Internazionale Matematico Estivo 1970

van Dantzig, D. On the phenomenological thermodynamics of moving matter. Physica 6, 673704 (1939)

Walton, R.: Hamiltonian dynamics of a self-gravitating isentropic perfect fluid, Thesis, Stanford University 1979

Walton, R.: [1980]:A new Hamiltonian formalism for general relativistic perfect fluids (unpublished).

Communicated by S.-T. Yau

Received May 30, 1984 
